\title{
Hacia un censo completo unificado de los ejemplares conservados de Celestina (I): ejemplares de ediciones en castellano localizables en línea ${ }^{1}$
}

\author{
Amaranta Saguar García \\ Universidad Complutense
}

\section{RESUMEN}

En una época en la que las bibliotecas no dejan de volcar nuevos catálogos en la red y de actualizar los antiguos, en la que los catálogos colectivos regionales no dejan de sacar a la luz y de hacer accesibles colecciones que de otra manera pasarían desapercibidas para los investigadores, en la que los catálogos y metacatálogos digitales permiten consultar de una sola vez decenas de fondos repartidos por el mundo, el redescubrimiento de ejemplares de Celestina perdidos o no consignados hasta el momento no debería sorprender a nadie. Estas especiales circunstancias han hecho que mantener un censo completo y actualizado de ejemplares localizados de Celestina requiera la revisión periódica de dichos recursos digitales con el objetivo de incorporar nuevos ejemplares, corroborar la existencia de otros y corregir los posibles ejemplares fantasma de versiones anteriores. El presente trabajo quiere ofrecer un punto de partida para esta revisión periódica reuniendo por escrito y en un mismo lugar todos los ejemplares conservados de ediciones en castellano de Celestina anteriores a su prohibición inquisitorial localizables en línea a 1 de noviembre de 2020.

Palabras Clave: Censo de ejemplares; Censo de ediciones

Towards an unified complete census of the surviving copies of Celestina (I): on-line retrievable copies of editions in Spanish

ABSTRACT

In a time in which libraries keep uploading new catalogues online and updating the old ones, in which regional union catalogues do not stop bringing to light and

1.- Este artículo forma parte de las tareas preliminares del proyecto La tradición iconográfica de "Celestina": materialidad y recepción de las ediciones ilustradas en la Edad Moderna (IcoCel), dentro del Programa de Atracción de Talento de la Comunidad de Madrid (2018-T1/HUM-11717), y de los materiales de apoyo para el proyecto Parnaseo. Servidor Web de Literatura Española, (FFI2017-82588-P AEI/FEDER, UE). 
making accessible collections that, otherwise, would go unnoticed by researchers, in which digital catalogues and metacatalogues allow looking up several collections distributed all over the world at the same time, rediscovering copies of Celestina lost or not recorded until now should not surprise anyone. These special circumstances have caused that maintaining a complete and up-to-date census of surviving copies of Celestina requires revising the above mentioned digital resources periodically, with the purpose of adding new copies, confirming the existence of others, and correcting the potential phantom copies of the previous versions. This work aims to offer a starting point for a periodical revision of these sources by means of bringing together in writing and at the same place all the online traceable copies of editions in Spanish printed prior to its Inquisitorial prohibition, as of the $1^{\text {st }}$ of November 2020.

Keywords: Census of Copies; Census of Editions

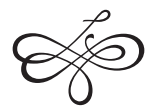

Este censo nace con la certeza de que no tardará en quedar obsoleto. Puede incluso que, mientras se publica, los catálogos y los repertorios digitales de los que depende cambien, se actualicen, y las observaciones de las próximas páginas dejen de ser pertinentes ${ }^{2}$. $\bigcirc$ puede que lo hagan a raíz de la publicación de este trabajo, si deciden incorporar las correcciones y las ampliaciones que en él se sugieren. $\bigcirc$ nunca, si de repente desaparece la financiación para su mantenimiento. En cualquier caso, la flexibilidad y la incierta supervivencia de los recursos en línea hace que cualquier revisión de los mismos esté necesariamente ceñida a un estado concreto, limitado en el tiempo, que, en lo concerniente a estas líneas, es el 1 de noviembre de $2020^{3}$.

2.- Estas palabras han resultado proféticas en cuanto que, mientras daba forma a este censo, el Universal Short Title Catalogue (USTC) ha implementado un formulario para la comunicación de errores que ya ha tenido algunas consecuencias para algunas de las entradas aquí reseñadas. Iberian Books (IB) ha corregido algunas de las erratas que comenté a través de Twitter y la Bibliografía Española de Textos Antiguos (BETA) y el Catálogo de Obras Medievales Impresas en Castellano (COMEDIC) han revisado y actualizado en algunos casos sus listados de ejemplares y ediciones en función a la información que les he ido haciendo llegar mientras progresaba en mi búsqueda, si bien aún hay diferencias entre sus censos y este que propongo.

3.- En realidad este censo se cerró en los primeros días de enero de 2020, sin embargo, puesto que Celestinesca aparece a finales de año, se decidió dejar pasar el tiempo y hacerle una revisión general antes de prepararlo para publicar, para que estuviese más actualizado. En este tiempo, especialmente BETA se ha beneficiado de una copia de una versión anterior de este mismo censo, que ha resultado sobre todo en la creación de doce nuevos identificadores para ediciones que hasta ahora no estaban consignadas (BETA manid 6177, 6178, 6179, 6180, $6181,6182,6183,6184,6185,6186,6187$ y 6188) y que, en el momento de escribir esta nota, no incluían aún información sobre los ejemplares conservados. 
Celestina es un texto con toda una tradición de pesquisas bibliográficas que, repertorios no específicos e inventarios aparte, por primera vez cristalizaron en el ensayo de catálogo cronológico y la reseña sobre las principales traducciones de Krapf (1900a; 1900b), con 79 (+1) entradas para las ediciones en castellano hasta 1900 y 36 para las traducciones. Le siguió la contribución de Givanel Mas (1919), con 113 (+1) entradas para las ediciones en castellano hasta 1919 y 56 para las traducciones. También les dedicó una sección de su bibliografía Cornejo (1977), con 151 entradas para las ediciones en castellano hasta 1970 y 58 para las traducciones.

Además de no distinguir entre ediciones antiguas y ediciones modernas, y a veces, incluso, entre versiones originales en castellano y traducciones, los repertorios mencionados admiten toda una serie de ediciones fantasma, hipotéticas y desconocidas pero potencialmente auténticas que multiplican los registros. Por eso resulta tan importante la lista de ediciones anteriores a 1650 con ejemplares conservados conocidos de la edición crítica de Celestina de Lobera et al. (Rojas y antiguo autor 2000) ${ }^{4}$, cuyas 82 entradas, 81 para las ediciones en castellano y 1 para la princeps de la traducción italiana, incluida exclusivamente debido a su importancia para demostrar la existencia de una edición de la Tragicomedia anterior a la de la del ejemplar más antiguo conservado, se han tenido por las únicas ediciones supervivientes confirmadas hasta que, hace relativamente poco, y gracias sobre todo a los esfuerzos de los colaboradores del Catálogo de Obras Medievales Impresas en Castellano (COMEDIC) y la Bibliografía Española de Textos Antiguos (BETA), pero también a los de Iberian Books (IB) y el Universal Short Title Catalogue (USTC), y a los de investigadores como Paolini (2013; 2010) o Griffin (2013), han empezado a salir a la luz ejemplares de ediciones desconocidas y de ediciones que se tenían por perdidas.

En los veinte años transcurridos desde la publicación de dicha lista se han localizado ejemplares de seis ediciones que entonces eran desconocidas: Sevilla, [Juan Varela de Salamanca], 1502 [pero 1514-1517], localizada por Ottavio Di Camillo en la Biblioteca Nazionale Vittorio Emanuele III de Nápoles (Fernández Valladares 2019); Sevilla, [Jacobo Cromberger], 1502 [pero 1516, antes de abril], encontrado por Charles Faulhaber en la Universitäts- und Forschungsbibliothek Erfurt/Gotha (Martín Abad 2017); Sevilla, herederos de Juan Cromberger, 12 de noviembre de 1543, descubierto hace unos años en la Bibliothèque Mazarine (Griffin 2013: 704-706); Zaragoza, Pedro Bernuz, 22 de noviembre de 1554, dado a conocer por el bibliófilo Alfonso Fernández González en un facsímil reciente (Rojas 2018); Sevilla, Alonso de la Barrera, 1569, hallado en la biblioteca municipal de Nantes, y Salamanca, Pedro Lasso Vaca, 1573, que había pasado inadver-

4.- Cito por la edición crítica de Lobera et al. (Rojas y antiguo autor 2000) y no por la edición digital de Botta (2001) debido a que la versión impresa ha sido la más difundida, a pesar de que la labor de la estudiosa italiana, desvinculada de la edición crítica por diferencias de criterio editorial poco antes de su publicación, resultó fundamental para su elaboración. 
tido hasta ahora en la Staatsbibliothek Voralberg (Lacarra 2019: 238). Además, se ha localizado en la Biblioteca comunal Valentiniana de Camerino un ejemplar de una edición [Sevilla], Jacobo y Juan Cromberger, noviembre de 1525, que no es idéntico al de la British Library con los mismos datos de impresión (Faulhaber 2020), por lo que actualmente se discute si se trata de una emisión de la edición conocida (postura de BETA) o de una edición desconocida (postura de COMEDIC que, por razones más prácticas que bibliográficas, seguiré en este trabajo, puesto que no me detendré en los problemas de emisión y estado, que quedan así pendientes para futuras actualizaciones).

Asimismo, también se ha desestimado la existencia de algunas ediciones fantasma incluidas en la lista de la edición crítica de Lobera et al. Así, la entrada $\mathrm{Amb}_{91}$ del repertorio, que se corresponde con una edición [Leiden], Oficina Plantiniana, 1591, con total seguridad responde a una errata por la edición [Leiden], Oficina Plantiniana, 1599, de la que la Van Pelt Library de la Universidad de Pensilvania sí tiene un ejemplar bajo la signatura 868 R63C 1599, mientras que la entrada Ven 36 resulta de la confusión entre la edición de la Segunda Celestina de Venecia, Stefano Nicolini da Sabio, 1536, y la de la Tragicomedia de Venecia, Stefano Nicolini da Sabio, 10 de julio de $1534^{5}$. Restadas estas dos ediciones de las 82 (-1) iniciales de la edición crítica de Lobera et al. y sumados los 6 nuevos descubrimientos vistos más arriba y 2 ediciones más que, tal vez por descuido, se quedaron fuera de la lista (Barcelona, Claudio Bornat, 1566, y Valencia, Juan Navarro, 1575), a 1 de noviembre de 2020 el número de ediciones antiguas de Celestina en castellano con ejemplares conservados localizados es de 87; 89 si, como hace COMEDIC, se tiene en cuenta la edición Alcalá de Henares, Juan de Villanueva, 1570-1571 (COMEDIC 63), preservada en un estado tan ínfimo (apenas medio pliego sin terminar de imprimir en las hojas de guarda de un ejemplar de otro texto que nada tiene que ver) que solo sirve a efectos cuantitativos, así como la de [Sevilla], Jacobo y Juan Cromberger, noviembre de 1525 de la Biblioteca comunal Valentiniana de Camerino (COMEDIC 18) ${ }^{6}$.

5.- No solo la lista de la edición crítica de Lobera et al. se hace eco de esta edición fantasma, sino también BETA (manid 5909). Es probable que en este error tenga que ver la circunstancia de que, hasta el 23 de enero de 2020, la Bayerische Staatsbibliothek tenía un ejemplar de la Segunda Celestina catalogado como de la Tragicomedia bajo la signatura Res/P.o.hisp. 195, que a día de hoy el amable personal de la biblioteca ha revisado y corregido a petición de los investigadores. El ejemplar de la Biblioteca Augusta Vaticana consignado por la edición de Lobera et al. hace referencia también a uno de la Segunda Celestina de Venecia, Stefano Nicolini da Sabio, 1536, con signatura R.G.Lett.Est.V.2516(int.2).

6.- Frente a la lista de la edición crítica de Lobera et al., limitada en principio a ediciones conocidas con ejemplares conservados, no obstante la edición fantasma mencionada en la nota anterior, los principales repertorios en línea que se ocupan de las ediciones de Celestina, es decir, COMEDIC, BETA, IB y el USTC, admiten ediciones de las que no se conservan ejemplares pero a las que sí se les concede credibilidad. Así, COMEDIC consigna las edicio- 
Animada por los recientes hallazgos de Di Camillo, Faulhaber, Fernández Valladares, Martín Abad y Neil Harris (Faulhaber 2020; Martín Abad 2017; Fernández Valladares 2019), y del equipo de COMEDIC (Lacarra 2019: 238), y sabedora de que los catálogos y metacatálogos en línea están en continuo proceso de mejora y actualización, decidí realizar un rastreo sistemático de los ejemplares supervivientes de Celestina localizables a través de la red con la finalidad de unificar, completar y actualizar los listados de ejemplares conservados recopilados por COMEDIC, BETA, IB y el USTC, así como de identificar sus posibles errores. Para ser fiel al carácter digital de mi objetivo, me propuse, además, localizar los enlaces permanentes a las entradas de los catálogos en línea pertinentes para los ejemplares ya consignados, además de las signaturas actuales de los ejemplares, a veces ausentes u obsoletas en los repertorios que me han precedido. Como resultado adicional, recopilé también todas las digitalizaciones que fueron apareciendo.

Partiendo de las 89 ediciones de existencia confirmada, extraje, comparé y combiné en una única lista los ejemplares conservados recogidos en

nes ¿[Salamanca], s.i., [1500]? (COMEDIC 3) y ¿Alcalá de Henares, Hernán Ramírez, 1594? (COMEDIC 78), la cual también recogen IB (76579) y el USTC (348204). Por su parte, BETA, IB y el USTC coinciden en incluir ¿Sevilla, s.i., 1504? (BETA manid 5866, IB 92254, USTC 349838), ¿Sevilla, s.i., 1534? (BETA manid 6031, IB 92337, USTC 349917), ¿Sevilla, s.i., 1539? (BETA manid 6026, IB 92361, USTC 350726), ¿Salamanca, s.i., 1552? (BETA manid 6027, IB 77531, USTC 348973), ¿Salamanca, herederos de Juan de Junta, 1558? (BETA manid 6028, IB 77562, USTC 349004), ¿[Leiden], Oficina Plantiniana, 1585? (BETA manid 6022, IB 76888, USTC 441603), ¿Alcalá de Henares, Hernán Ramírez, 1590? (BETA manid 6023, IB 76890, USTC 348392), ¿[Leiden], Oficina Plantiniana, 1590? (BETA manid 6030, IB 76889, USTC 441604) y ¿Sevilla, Rodrigo de Cabrera, 1599? (BETA manid 6024, IB 76892, USTC 348393). Asimismo, consignan como edición sin ejemplares la de Sevilla, Alonso de la Barrera, 1569 (IB 76887, USTC 348389, BETA manid 6029), siendo COMEDIC (59) el único repertorio que se hace eco del redescubrimiento del ejemplar de Nantes. El USTC e IB comparten, además, una edición errónea - Venecia, Juan Batista Pedrezano, 1521 (IB 65084, USTC 344230), que sin lugar a duda es una errata por la edición de 1531 (no de 1631, como aparece en IB)_, a la que el USTC añade también las ediciones inexistentes Amberes, Martín Nucio, 1550 (USTC 442809), que además aparece registrada como texto en latín, y Amberes, Philippo Nucio, 1563 (USTC 440319), que es una errata por la edición de 1568. Los cuatro repertorios difieren también en el número de ediciones diferentes y en la existencia o no de ejemplares localizados: 80 ediciones para COMEDIC (82 si se añaden las ediciones sin ejemplares localizados) antes de 1600 y 77 para BETA (89 con las ediciones con ejemplares conocidos pero que aún no consignan ejemplares de la nota 2, 99 con las ediciones sin ejemplares localizados y de existencia hipotética, 100 con la edición fantasma Venecia, Stefano Nicolini da Sabio, 1536, y 102 si sumamos, además, los manuscritos de Palacio - BETA manid 4776- y Celestina comentada -BETA manid 5523-, que BETA también consigna), 81 - si bien dos de las ediciones tienen entradas duplicadas- para IB (91 con las ediciones sin ejemplares localizados y 92 con la edición fantasma Venecia, Juan Batista Pedrezano, 1521), y 82 - pero 85 si sumamos las ediciones bilingües español-francés consignadas como ediciones exclusivamente en francés (USTC 6812310 y 6813067) y la edición erróneamente descrita como en latín (USTC 442809)_ - para el USTC (93 con las ediciones sin ejemplares localizados y 96 con las ediciones fantasma Venecia, Juan Batista Pedrezano, 1521; Amberes, Martín Nucio, 1550, y Amberes, Philippo Nucio, 1563) antes de 1650. 
COMEDIC, BETA, IB y el USTC — también el Incunabula Short Title Catalogue (ISTC) y el Gesamtkatalog der Wiegendrucke (GW) para las ediciones anteriores a 1500-, y para cada una de ellas y comprobé individualmente cada ejemplar consignado en el catálogo en línea de la biblioteca correspondiente. En el caso de los ejemplares que no figuraban en el catálogo en línea que les correspondía o localizados en colecciones privadas o sin catálogo digital, los busqué en los catálogos colectivos nacionales correspondientes. Aproveché cada búsqueda para rastrear cada catálogo consultado, particular o colectivo, en busca de ejemplares adicionales no consignados en mis fuentes. Finalmente, cuando fue necesario, recurrí -no siempre con éxito- a fuentes escritas y/o a comunicarme directamente con el personal de las bibliotecas relevantes, especialmente cuando COMEDIC, BETA, IB o el USTC consignaban un ejemplar pero no había rastro de este en línea. Desde aquí quiero agradecer su inestimable ayuda al personal de todas las bibliotecas con las que me he puesto en contacto, ya que sin su trabajo este censo estaría mucho más incompleto.

El siguiente paso fue la búsqueda en masa de ejemplares no localizados de las ediciones conocidas a través del metacatálogo Karlsruher Virtueller Katalog (KVK), que permite consultar de una sola vez los catálogos colectivos de varios países (Alemania, Austria, Bélgica, Canadá, España, Estonia, Finlandia, Francia, Israel, Italia, Letonia, Luxemburgo, Noruega, Polonia, Portugal, Reino Unido, Suecia y Suiza), de varias bibliotecas nacionales (además de las de los países anteriores, las de Australia, Dinamarca, Holanda, Hungría, República Checa y Rusia) y WorldCat. A veces, comprobé incluso la información por duplicado accediendo personalmente a los catálogos colectivos nacionales, en especial el Catálogo Colectivo del Patrimonio Bibliográfico Español (CCPB), el Catalogue Collectif de France (CCFr), el Catalogo del Servizio Bibliotecario Nazionale (ICCU SBN), el Library Hub Discover (LHD, antiguo COPAC), la Base Nacional de Dados Bibliográficos (PORBA$\mathrm{SE}$ ), el Union Catalogue of Belgian Libraries (UniCat) y los catálogos colectivos alemanes Bibliotheksverbund Bayern (BVB), Gemeinsamer Bibliotheksverbund (GBV), Hessisches Bibliotheksinformationssystem (HeBIS), Kooperativer Bibliotheksverbund Berlin-Brandenburg (KOPV), Nordrhein-Westfälischer Bibliotheksverbund (HBZ) y Südwestdeutscher Bibliotheksverbund (SWB).

Por último, repetí el proceso del párrafo anterior pero con búsquedas libres, no ceñidas a ediciones conocidas de Celestina. Limitando la búsqueda al rango cronológico entre los años 1500 (con Infantes 2007, fecha más probable de la princeps de la Comedia) y 1792 (fecha de la prohibición inquisitorial de Celestina), utilicé las cadenas de búsqueda "Celestina", "Tragicomedia de Calisto y Melibea», "Tragicomedia de Calisto», "Calisto y Melibea», "Calisto" $\mathrm{y}$ "Melibea». También busqué por "Fernando de Rojas», «Rojas, Fernando de» y «de Rojas, Fernando». 


\section{Primer resultado: ediciones no conocidas}

El rastreo de ejemplares conservados de ediciones de Celestina en castellano anteriores a 1600 a través de los catálogos y metacatálogos arriba mencionados no ha añadido ninguna nueva edición a las 89 confirmadas. La pista de una potencial edición desconocida Sevilla, Francisco Pérez para Alonso de Mata, s. d. [pero 1584-1594], se pierde en la Biblioteca Oliveriana de Pesaro, cuyo catálogo consigna un ejemplar de la misma con la signatura BP 07-05-56 que, por desgracia, hoy día se encuentra perdi$\mathrm{do}^{7}$. La ficha ubica la impresión en el siglo XviII, precisamente el único del que no se conocen ediciones de Celestina en castellano, por lo que resulta mucho más probable que el Francisco Pérez a cargo de esta edición no sea el "Libraio a Madrid in calle de Atocha sec. 18» que señala el catálogo digital, sino el Francisco Pérez que estuvo activo en Sevilla entre 1584 y 1609 (CERL Thesaurus: cni00043912; Castillejo Benavente 2019: n II.35; Álvarez Márquez 2009: I, 180-182; Escudero y Perosso 1894: n XLII), mientras que el Alonso de Mata que financia la edición sería el mismo que costeó la del Amadís de Gaula de Sevilla, Fernando Díaz, 1586, documentado en Sevilla entre 1566 y 1594 (Álvarez Márquez 2009: II.2, 64-78; Álvarez Márquez 2007: passim, pero sobre todo 182-189).

Una obra como Celestina encaja bien con la orientación comercial tanto de Francisco Pérez como de Alonso de Mata, especializados, cada uno en su papel de impresor y de comerciante de libros, en obras prácticas y de consumo con las ventas aseguradas. Cronológicamente, además, el período 1584-1594 en el que tanto Francisco Pérez como Alonso de la Mata están simultáneamente en activo en Sevilla encaja bien en la sucesión de ediciones sevillanas de la segunda mitad del siglo: Sebastián Trujillo, 1562; Alonso de la Barrera, 1569; Alonso Picardo para Pedro Miguel, 1575; Alonso de la Barrera para Luis Torrero, 1582, y Sevilla, Fernando de Lara, 1596. Estas están espaciadas entre sí unos 6-7 años, a excepción de las dos últimas, a las que separan 14 años y entre las cuales habría habido lugar para esta edición (¿en torno a 1589?). Sin embargo, por verosímil que resulte su existencia, mientras no aparezca el ejemplar correspondiente de la Biblioteca Oliveriana u otro no deja de ser hipotética.

\section{Segundo resultado: ejemplares no consignados en los repertorios consultados}

El resultado de estas pesquisas han sido 28 ejemplares no recogidos en ninguno de los cuatro grandes repertorios (COMEDIC, BETA, IB y el

7.- Agradezco al personal de la Biblioteca Oliveriana su ayuda a la hora de intentar localizar el ejemplar. 
USTC) para las ediciones conocidas hasta 1600: 1 para Venecia, Stefano da Sabio, 10 de julio de 1534; 1 para Amberes, Guillaume du Mont, 28 de junio de 1539; 3 para Venecia, Gabriel Giolito de Ferrariis y sus hermanos, 20 de enero de 1553; 2 para Venecia, Gabriel Giolito de Ferrariis y sus hermanos, 1556 [20 de enero de 1553]; 1 para Amberes, viuda de Martín Nucio, 1558; 4 para Amberes, Philippo Nucio, 1568; 1 para [Leiden], Oficina Plantiniana, 1595, y 14 para [Leiden], Oficina Plantiniana, 1599. Incluidos estos, el total de ejemplares localizados anteriores a 1600 asciende a $378^{8}$.

Para el siglo XVII, se han añadido 2 ejemplares a los localizados de la edición Madrid, Andrés Sánchez para Miguel Martínez, 1601; 1 a los de la edición Juan de la Cuesta, 1619; 2 a los de la edición Milán, Juan Baptista Bidelo, 1622; 1 a los de la edición Madrid, viuda de Alonso Martín, 1631 [1632]; 1 a los de la edición Pamplona, Carlos Labayén, 1633; 3 a los de la edición Ruan, Charles Osmont, 1633, y 5 a los de la edición Ruan, Charles Osmont, 1634, lo que hace un total de 15 ejemplares adicionales. Sumados a los ya conocidos, son 94 los ejemplares seiscentistas.

Todo junto, hablamos de 476 ejemplares supervivientes de ediciones de Celestina en castellano localizados. Sin embargo, lo más interesante de estas cifras no es tanto su monto como, por un lado, lo que permiten predecir sobre futuros descubrimientos de nuevos ejemplares y de nuevas ediciones celestinescos, por otro, las hipótesis que permiten formular sobre el éxito editorial y el público lector de las ediciones en castellano de Celestina. En cuanto a lo primero, resulta especialmente significativo que una parte importante de los ejemplares nuevamente localizados provengan de bibliotecas municipales, universidades no muy grandes y colecciones vinculadas a la Iglesia que, a menos que los investigadores tuvieran indicios claros que apuntaran hacia ellas, no se han tenido en cuenta en los rastreos sistemáticos anteriores, ya sea por la orientación de sus fondos, ya por cuestiones de accesibilidad. Con la digitalización de sus catálogos y/o su incorporación a los catálogos colectivos de su región la distinción práctica entre fondos susceptibles de tener ejemplares de Celestina y fondos que no, destinada en su día a optimizar los esfuerzos de los investigadores, ha perdido todo el sentido ante la posibilidad de, con una sola búsqueda informatizada y desde un único lugar, consultar todas las colecciones de una vez. En consecuencia, en la actualidad estamos asistiendo -y seguiremos haciéndolo en el futuro inmediato, a medida que se digitalicen más y más catálogos, se establezcan más y más

8.- COMEDIC, el repertorio inicialmente más completo para su rango cronológico, recoge 339 ejemplares hasta 1600, con alguno duplicado, mientras que BETA, IB y el USTC recogen, respectivamente, 318, 375 y 418 ejemplares hasta el año 1650, descontadas las reproducciones (que tanto IB como el USTC consignan entre los ejemplares originales, mejor identificados en IB que en el USTC, donde no siempre se indica que se trata de reproducciones) y los ejemplares cuyo paradero se desconoce (ejemplares subastados, ejemplares perdidos, etc.), pero no los duplicados ni los errores. 
catálogos colectivos y los ya existentes estén más y más completos-a la localización de ejemplares de Celestina en fondos en los que de ninguna manera era previsible encontrarlos.

Asimismo, resulta significativo que un número nada despreciable de ejemplares haya reaparecido en regiones cuya lengua queda fuera de la triada español, inglés y una segunda lengua romance, con predominio del italiano y del francés, que -y aquí hablo desde un punto de vista subjetivo- parece proliferar entre los celestinistas. La falta de familiaridad con lenguas distintas de estas, unida a lo poco probable que puede llegar a parecer que un texto en castellano acabe en una región en la que ni tan siquiera se habla una lengua romance, explicaría que los catálogos de dichas partes de mundo hayan sido mucho menos explorados. Solo así puedo explicarme el alto porcentaje de ejemplares localizados nuevamente en países germanoparlantes, ya que los catálogos colectivos alemanes llevan funcionando el tiempo suficiente como para tener que haber sido tenidos en cuenta para los rastreos de ejemplares que han precedido a este. A la barrera lingüística podría sumarse, además, cierto eurocentrismo occidental inconsciente, por el cual los catálogos de las regiones no consideradas como propiamente occidentales han recibido menor atención, lo que podría justificar que tres de los nuevos ejemplares localizados lo hayan sido en Polonia y Rusia. En cualquier caso, resulta evidente que un rastreo de las bibliotecas fuera de la «zona de confort» lingüística de los celestinistas y del foco europeo occidental necesariamente tiene que desembocar en la localización de nuevos ejemplares en los próximos años, probablemente también propiciada por la proliferación de nuevos catálogos digitales. No en vano, el uso de estándares de catalogación y su formulación como metadatos estandarizados hacen que la barrera lingüística deje de suponer un obstáculo a la hora de lanzar búsquedas informatizadas, más allá de la posible transcripción del título del texto o del nombre del autor a la lengua local, y aun así para esto también se está trabajando en unificar criterios a través del Fichero de Autoridades Virtual Internacional (VIAF).

El reparto cronológico de los ejemplares nuevamente localizados permite, igualmente, hacer una última predicción sobre el descubrimiento de nuevos ejemplares de Celestina en castellano. Frente a los apenas dos ejemplares añadidos para el período entre 1500 y 1550, los 25 del período de 1550 a 1600 y los 15 de entre 1600 y 1650 determinan la tendencia que probablemente se siga en los próximos años. Así, seguirán apareciendo con relativa frecuencia ejemplares de las ediciones de la segunda mitad del siglo Xvi, época en la que Celestina es ya un texto más que consolidado, poco susceptible a novedades editoriales porque su venta está asegurada sin necesidad de recurrir a estrategias de marketing. Es una obra prácticamente de stock para los impresores y los libreros del momento, con tiradas ni grandes ni pequeñas, sino calculadas para satisfacer un gra- 
do más o menos constante de demanda, y en formato de bolsillo, por lo tanto, un libro muy extendido y no demasiado vistoso ni valioso, no el best-seller que fue pero tampoco una obra ignorada, que encaja en las bibliotecas de todo tipo, gracias a lo cual los ejemplares han multiplicado sus posibilidades de supervivencia. Puede, incluso, que aparezca alguna edición desconocida más para este período, a juzgar por los indicios que apuntan a una edición Sevilla, Francisco Pérez para Alonso de Mata, [1584-1594], vistos en el apartado 1.

Asimismo, es posible que aparezcan nuevos ejemplares para las ediciones seiscentistas, pero en menor proporción, debido a que la orden de expurgo de 1632 no solo truncó la carrera editorial de la obra en castellano, cuyo ritmo estaba, además, en claro descenso desde el último cuarto del siglo XVI, sino que con casi total probabilidad provocó la pérdida de ejemplares de ediciones más o menos cercanas en el tiempo a manos de lectores con menos paciencia que los que se esforzaron en censurar sus ejemplares sin expurgar (véase, por ejemplo, el de la Biblioteca Nacional de España con signatura R/31686), y de libreros escrupulosos lastrados con un material que no podían vender. Por esta misma razón es también muy probable que los nuevos ejemplares que aparezcan pertenezcan a alguna de las ediciones bilingües posteriores al expurgo impresas en Francia, que parecen haber vivido más al margen de la censura inquisitorial ${ }^{9}$.

Finalmente, parece menos probable que aparezcan nuevos ejemplares de las ediciones conocidas para la primera mitad del siglo XVI que el que lo hagan ejemplares únicos de ediciones desconocidas ${ }^{10}$. No solo así lo evidencian los recientes descubrimientos (cuatro ejemplares únicos de ediciones desconocidas anteriores a 1550 frente a dos ejemplares de ediciones conocidas de la misma época), sino que también lo corrobora el estado mínimo en el que se conserva la mayoría de las ediciones anteriores a 1550: de 40 ediciones, 25 se conocen a partir de un único ejemplar, frente a las 19 de 40 del período 1550-1600. De hecho, lo que sabemos de la historia editorial de Celestina en su primer medio siglo de vida invita a pensar en un número mucho mayor de ediciones que en la mitad siguiente, frente al aparente equilibro entre las dos mitades del censo actual. En consecuencia, los ejemplares supervivientes anteriores a 1550 que aparezcan en los próximos años probablemente pertenezcan a ediciones desconocidas. Además, aparecerán muy raramente, en cuanto que su supervivencia ha sido más difícil, bien por tratarse de objetos de relativo valor, proclives por lo tanto a expolios de todo tipo y con una dis-

9.- Según Palau (1950: n 51169), la edición Pamplona, Carlos Labayén, 1633, con casi total seguridad fue también publicada por Charles Osmont en Ruan, y comercializada con portada falsa.

10.- El descubrimiento el pasado febrero de un ejemplar de una potencial nueva edición [Sevilla], Jacobo y Juan Cromberger, noviembre de 1525, en la Biblioteca comunale Valentiniana de Camerino parece confirmar esta intuición (véase también la nota 17). 
tribución social y cultural que limita los lugares donde pudieron conservarse; bien por haber sido desplazados por ejemplares de ediciones más modernas en las colecciones de sus poseedores; bien por más años de uso continuado o por un uso más intenso relacionado con el éxito de la obra.

\section{Censo de ejemplares de Celestina a 1 de noviembre de 2020}

Consciente de que, debido a su dependencia de los medios digitales $-\mathrm{y}$, además, de medios digitales muy concretos-y la deliberada omisión de los medios tradicionales en papel, mi revisión no es tan exhaustiva como sería deseable para poder establecer un censo real de ejemplares de ediciones en castellano de Celestina, ofrezco, no obstante, a continuación una lista de los ejemplares cuya existencia está apoyada por la correspondiente entrada en el catálogo digital de la biblioteca en la que se conserva o, en su defecto, en alguno de los catálogos colectivos arriba mencionados o por la autoridad de BETA, COMEDIC, IB o el USTC (con los comentarios pertinentes). Cuando la información de estos repertorios me ha parecido dudosa u obsoleta, especialmente cuando no he encontrado otra fuente actualizada fuera de estos que apoye la presencia de un ejemplar en tal o cual colección, he procedido a contactar directamente con las bibliotecas, pero no de manera sistemática, por lo que puedo haber reproducido informaciones erróneas. Igualmente, puedo haber introducido errores en lo que a la asignación de determinados ejemplares a determinadas ediciones se refiere, ya que he trabajado con los registros catalográficos y solo en contadas ocasiones con los ejemplares. A todo esto hay que sumar la inevitable falibilidad humana, por lo que pido disculpas por adelantado por los errores que pueda haber introducido yo misma en este primer intento de censo completo de ejemplares supervivientes de ediciones en castellano de Celestina, y animo a todos los celestinistas a revisarlo, corregirlo y completarlo en futuras ocasiones.

Cada entrada del censo está identificada por un número, al que siguen, en este orden, el lugar de impresión, el impresor y, en caso de haberlo, el financiador de la edición, y la fecha. Después de esta información general se incluye, entre paréntesis, el código abreviado que propongo usar para futuros trabajos sobre las ediciones de Celestina, inspirado en el que utilizaran Lobera et al. en su edición crítica de 2000: a las primeras letras del lugar de impresión les sigue el año o el rango cronológico, con letras para diferenciar las ediciones que coinciden. Se salen de esta norma la edición en verso de Sedeño que, por su excepcionalidad, recibe el código Sed, y las ediciones con pie de imprenta de la Oficina Plantiniana, en las que sistemáticamente se omite el lugar de impresión (Leiden para unos especialistas, Amberes para otros) y que, en consecuencia, se sustituye por la abreviatura de la imprenta Plant. Tras este paréntesis indico el es- 
tado redaccional de la edición con las siglas CCM para la Comedia en 16 autos, TCM para la Tragicomedia en 21 autos, TR para la Tragicomedia en 22 autos, EXP para la Tragicomedia expurgada y BIL para las versiones bilingües español-francés. A continuación, tras un salto de línea, ofrezco información sobre los principales catálogos digitales que consignan la edición, incluidos los identificadores pertinentes y, de haberlos localizado, los enlaces permanentes a los mismos. Finalmente, incluyo la información sobre los ejemplares localizados repartida en tres secciones: «Ejemplares localizados con entrada en catálogo en línea», "Ejemplares localizados sin entrada en catálogo en línea» y, por último, "Ejemplares sin asignar, sin localizar o perdidos", que no se tienen en cuenta para ninguno de los cómputos de ejemplares hechos más arriba. Para cada sección añado, entre paréntesis, el número de ejemplares consignados $y$, para cada ejemplar, proporciono, en este orden, la ciudad, la biblioteca, la signatura enlazada a la entrada pertinente del catálogo en línea (si se dispone de ambos, si no, solamente se proporciona la signatura, sin enlace, o no se proporcionan más detalles) y, entre paréntesis, cualquier comentario sobre el ejemplar que pueda ser de interés, incluidos los enlaces a digitalizaciones. En lo que con toda seguridad es una decisión antibibliográfica, no distingo por colección ni por biblioteca en los casos de bibliotecas universitarias o bibliotecas con varias sedes, en cuanto que los catálogos enlazados y/o citados proporcionan información al respecto o es información sencilla de recabar. Por otra parte, cuando hay ejemplares sin asignar, sin localizar o perdidos conocidos únicamente a través de repertorios de bibliotecas privadas y catálogos de libreros o de subastas, pero sin vinculación conocida a una biblioteca en particular, proporciono la denominación por la que son conocidos - generalmente los nombres de sus antiguos poseedores- $y$, cuando lo hay, el identificador correspondiente en BETA, pertinentemente enlazado.

Combino en esta lista las informaciones de todas mis fuentes sin indicación de proveniencia más que en los casos en los que no se dispone de una entrada en un catálogo digital para probarlas. Asimismo, explico en nota las variaciones respecto a COMEDIC, BETA, IB y el USTC, y marco en cursiva los ejemplares que incorporo al censo y que, en el momento de cerrar este censo, no aparecen en ninguno de estos cuatro repertorios. 
1. [Burgos], Fadrique de Basilea, 1499 [pero ca. 1499-1501] (Bur1499): CCM

Principales repertorios en línea: IB (50538), ISTC (if00100000), GW (M38597), USTC (333587 y 767401)11, BETA (manid 2119), COMEDIC (1)

Ejemplares localizados con entrada en catálogo en línea (1):

- Nueva York, Hispanic Society of America, Inc 75 (ejemplar único, mútilo de la primera hoja y manipulado; facsímil digitalizado en la Biblioteca Virtual Miguel de Cervantes, Google Books y TeXTRed; próximamente también en el Portal Celestinesco de PARNASEO)

\section{Toledo, [Pedro Hagenbach], 1500 (Tol1500): CCM}

Principales repertorios en línea: IB (50571), ISTC (if00100100), GW (M38600), USTC (767612 y 333619), BETA (manid 2120), COMEDIC (2)

Ejemplares localizados sin entrada en catálogo en línea (1):

- Cologny, Fondation Martin Bodmer, Inc. 66 (ejemplar único)

\section{Sevilla, Estanislao Polono, 1501 (Sev1501): CCM}

Principales repertorios en línea: IB (65015), USTC (344185), BETA (manid 4607), COMEDIC (4)

Ejemplares localizados con entrada en catálogo en línea (1):

- París, Bibliothèque Nationale de France, Rés. Yg.63 (ejemplar único; digitalizado en Gallica)

\section{Zaragoza, [Jorge Coci], 1507 (Zar1507): TCM}

Principales repertorios en línea: IB (60154), USTC (341404), BETA (manid 3883), COMEDIC (5)

Ejemplares localizados con entrada en catálogo en línea (1):

- Madrid, Real Academia de la Historia, Inc. San Román 37 (ejemplar mútilo de las cuatro primeras hojas)

Ejemplares localizados sin entrada en catálogo en línea (1):

- Toledo, Biblioteca del Cigarral del Carmen (único ejemplar completo; colección privada, Botta e Infantes 1999)

11.- Puesto que el USTC se alimenta de catálogos preexistentes, no es extraño que genere más de una entrada para una misma edición si los datos de esta aparecen introducidos de manera diferente en sus fuentes, no hay consenso sobre la imprenta, la fecha y/o el lugar de impresión, o están mal catalogados. Esto también conduce a la duplicación de ejemplares y a la confusión entre copias originales y reproducciones (facsímiles, microfilms, digitalizaciones, etc.). Afortunadamente, desde hace algún tiempo el USTC incluye un formulario para comunicar errores. 
5. Toledo, [sucesor de Pedro Hagenbach], 1502 [pero 1510-1514] (Tol1502): TCM

Principales repertorios en línea: IB (63955), USTC (343237), BETA (manid 5223), COMEDIC (6)

Ejemplares localizados con entrada en catálogo en línea (1):

- Londres, British Library, C.20.b.9. (ejemplar único, mútilo de las primeras cinco hojas del cuadernillo $f$ )

6. Sevilla, Jacobo Cromberger], 1502 [pero ca. 1511 (Sev1502a): TCM

Principales repertorios en línea: IB (63956), USTC (343238), BETA (manid 4616), COMEDIC (7)

Ejemplares localizados con entrada en catálogo en línea (1):

- Londres, British Library, C.20.c.17. (ejemplar único)

7. Sevilla], Jacobo Cromberger], [1502, pero 1513-1515 (Sev1502b): TCM

Principales repertorios en línea: IB (65083), USTC (344229), BETA (manid 5224), COMEDIC (8)

Ejemplares localizados con entrada en catálogo en línea (1):

- Ann Arbor, University of Michigan, PQ6426 .A1 1502? (ejemplar único, mútilo de las hojas b1, b8, c1, c8, d2, h1 y h8; digitalizado en Hathi Trust $)^{12}$

8. Valencia, Juan Joffre, 21 de febrero de 1514 (Val1514): TCM

Principales repertorios en línea: IB (60155), USTC (341405), BETA (manid 4327), COMEDIC (10)

Ejemplares localizados con entrada en catálogo en línea (1):

- Madrid, Biblioteca Nacional de España, R/4870 (ejemplar único, digitalizado en la Biblioteca Virtual Miguel de Cervantes)

9. Sevilla, [Juan Varela de Salamanca], 1502 [pero ca. 1514-1517] (Sev1502c): TCM

Principales repertorios en línea: IB (98521), USTC (5046471), BETA (manid 5872), COMEDIC (9)

Ejemplares localizados sin entrada en catálogo en línea (1):

- Nápoles, Biblioteca Nazionale Vittorio Emanuele III, XXIIIC-31(1) (ejemplar único; Fernández Valladares 2019) 
10. Sevilla [pero Roma], [Marcello Silber], 1502 [pero 1515-1516] (Sev1502d): TCM

Principales repertorios en línea: IB (63957), USTC (343239)13, BETA (manid 5225), COMEDIC (11)

Ejemplares localizados con entrada en catálogo en línea (4):

- Boston, Boston Public Library, XD.170B.9 (ejemplar digitalizado en Archive.org)

- Buenos Aires, Biblioteca Nacional Mariano Moreno, 214 FD (ejemplar digitalizado en la Biblioteca Virtual Miguel de Cervantes y en BNMN)

- Londres, British Library, C.20.b.15. (mútilo de la última hoja, con todas las coplas finales, que según el catálogo es sustituida por una de un facsímil de la edición Sev1501)

- Tréveris, Stadtbibliothek, G 578: 2 an

Ejemplares localizados sin entrada en catálogo en línea (1):

- Madrid, Biblioteca Francisco de Zabálburu, 2:249-50 núm. 2277 (mútilo de las cinco últimas hojas, colección privada; CСРB)

Ejemplares sin asignar, sin localizar o perdidos (1):

- Ejemplar Heber-Soleinne-Taylor-Techener (BETA copid 3183)

11. Sevilla, [Jacobo Cromberger], 1502 [pero 1516, antes de abril] (Sev1502e): TCM

Principales repertorios en línea: IB (98522), USTC (5046472), BETA (manid 5905), COMEDIC (12)

Ejemplares localizados con entrada en catálogo en línea (1):

- Gotha, Universitäts- und Forschungsbibliothek Erfurt/Gotha, 03 - Lp. $8^{\circ} 00550$ (ejemplar único)

12. Valencia, Juan Joffré, 27 de marzo de 1518 (Val1518): TCM

Principales repertorios en línea: IB (63958), USTC (343240), BETA (manid 5805), COMEDIC (14)

Ejemplares localizados con entrada en catálogo en línea (1):

- Londres, British Library, C.64.d.4. (ejemplar único)

13. Sevilla, [Jacobo Cromberger], 1502 [pero 1518-1520] (Sev1502f): TCM

Principales repertorios en línea: IB (60149), USTC (341399), BETA (manid 4617), COMEDIC (13) 
Ejemplares localizados con entrada en catálogo en línea (1 $)^{14}$ :

- Madrid, Biblioteca Nacional de España, R/26575 (ejemplar único, cuaderno e encuadernado delante del cuaderno d; digitalizado en la Biblioteca Digital Hispánica)

\section{4. ¿Salamanca? [pero Roma], [Antonio de Blado para Antonio de Salamanca], 1502 [pero 1520] (Sal1502): TCM}

Principales repertorios en línea: IB (63959), USTC (343241) ${ }^{15}$, BETA (manid 5256), COMEDIC (15)

Ejemplares localizados con entrada en catálogo en línea (2):

- Londres, British Library, G.10224.

- Nueva York, Hispanic Society of America, PQ 6426 .A1 [ca. 1520] (ejemplar digitalizado en TeXTRed)

Ejemplares localizados sin entrada en catálogo en línea (1):

- Palma de Mallorca, biblioteca de Bartolomé March (colección privada; Infantes 2007)

15. Sevilla [pero Venecia], Juan Bautista Pedrezano], 1523 (Sev1523): TCM

Principales repertorios en línea: IB $(63960,63961)^{16}$, USTC (343242 y 343243$)^{17}$, BETA (manid 5634), COMEDIC (16)

Ejemplares localizados con entrada en catálogo en línea (12):

- Chantilly, Bibliothèque du Château de Chantilly, III-D-047

- Ciudad del Vaticano, Biblioteca Apostólica Vaticana, R.G.Lett. Est.V.2516(int.1) (incluye algunos folios manuscritos, no sé si en sustitución de páginas faltantes o no)

- Estrasburgo, Bibliothèque Nationale et Universitaire de Strasbourg, R.100.186,1

- Londres, British Library, C.63.e.8.

- Londres, British Library, C.20.a.13.(3.) (el cuadernillo L está defectuoso, pues la forma exterior se ha impreso en los dos lados del pliego y, por lo tanto, se repiten las páginas)

- Madrid, Biblioteca Nacional de España, R/30427

- Nueva York, Hispanic Society of America, PQ 6426 .A1 1523 (ejemplar digitalizado en TeXTRed)

14.- IB y el USTC consignan, además, un ejemplar en la Universidad de Michigan con la signatura Q 6426.A1 1502? (en realidad, PQ 6426 .A1 1502?), pero sin lugar a duda es una errata por el ejemplar único de la edición Sev1502b.

15.- El ejemplar de Richmond que consigna el USTC es una reproducción.

16.- Por las mismas razones que el USTC (nota 10), IB duplica en ocasiones los registros para sus ediciones, lo que a veces conlleva también la duplicación de ejemplares.

17.- El ejemplar con signatura R81088 consignado por el USTC de la Bibliothèque Nationale de France es una reproducción. 
- Oviedo, Universidad de Oviedo, CEA-057

- París, Bibliothèque Nationale de France, RESERVE 8-RE-6539 (ejemplar digitalizado en Gallica)

- Venecia, Fondazione Giorgio Cini, FOAN TES 128

- Viena, Österreichische Nationalbibliothek, *38.E.234 (ejemplar digitalizado en Austrian Books Online y Google Books)

- Washington, Library of Congress, Rosenwald 811, PQ6426 .A1 1523

Ejemplares localizados sin entrada en catálogo en línea (6):

- Broni, Collegiata de Broni, 128 (Busquets 1998: n 535)

- Milán, Biblioteca Pinacoteca Accademia Ambrosiana, S.Q.N.I.46 (Busquets 1998: n 535)

- Milán, Biblioteca Pinacoteca Accademia Ambrosiana, S.Q.N.I.47 (Busquets 1998: n 535 )

- Milán, Biblioteca Trivulziana dell'Archivio Storico-Civico, TRIV.L.1019/1 (Busquets 1998: n 535)

- Praga, Národní knihovna České republiky [Biblioteca Nacional de la República Checa] (Faulhaber 2015)

- Zaragoza, Biblioteca de Alfonso Fernández González (colección particular; COMEDIC)

Ejemplares sin asignar, sin localizar o perdidos (3):

- Ejemplar Foulché-Delbosc (BETA copid 8642)

- Ejemplar Heber-Soleinne-Taylor-Salvá-Heredia (BETA copid 4069)

- Ejemplar Seillère (BETA copid 4117)

\section{Barcelona, Carles Amorós, 1525 (Bar1525): TCM}

Principales repertorios en línea: IB (76883), USTC (348386), BETA (manid 5793), COMEDIC (17)

Ejemplares localizados con entrada en catálogo en línea (1):

- Barcelona, Biblioteca de Catalunya, Bon. 7-III-28 (ejemplar único; digitalizado en Google Books)

17. [Sevilla], Jacobo y Juan Cromberger, noviembre de 1525 (Sev1525a): TCM

Principales repertorios en línea: IB (63962), USTC (343244), BETA (manid 5806), COMEDIC (19)

Ejemplares localizados con entrada en catálogo en línea (1):

- Londres, British Library, G.10223. (ejemplar único)

18. [Sevilla], Jacobo y Juan Cromberger, noviembre de 1525 (Sev1525b): TCM

Principales repertorios en línea: IB (-), USTC (-), BETA (-), COMEDIC (18) 
Ejemplares localizados con entrada en catálogo en línea (1)

- Camerino, Biblioteca Comunale Valentiniana, Sec B. 926 (234) (ejemplar único)

19. Toledo, Remón de Petras, 23 de junio de 1526 (Tol1526): TR

Principales repertorios en línea: IB (63963), USTC (343245), BETA (manid 5803), COMEDIC (21)

Ejemplares localizados con entrada en catálogo en línea (1):

- Londres, British Library, C.63.c.24. (ejemplar único)

20. [Valladolid], [Nicolás Tierry], [ca. 1526-1540] (Vall1526-1540): TR

Principales repertorios en línea: IB (-), USTC (-), BETA (manid 5835), COMEDIC (20)

Ejemplares localizados con entrada en catálogo en línea (1):

- San Petesburgo, Росси́йская национа́льная библиоте́ка [Biblioteca Nacional de Rusia], 6.20.4.24 (ejemplar único, incompleto)

21. Sevilla, Jacobo y Juan Cromberger, marzo de 1528 (Sev1528): TCM

Principales repertorios en línea: IB (60157), USTC (341406), BETA (manid 5832), COMEDIC (22)

Ejemplares localizados con entrada en catálogo en línea (2):

- Madrid, Biblioteca Nacional de España, R/30275

- Nueva York, Hispanic Society of America, PQ 6426 .A1 1528 (ejemplar digitalizado en TeXTRed)

22. Valencia, Juan Viñao, 1529 (Val1529): TCM

Principales repertorios en línea: IB (63964), USTC (343246), BETA (manid 5804), COMEDIC (23)

Ejemplares localizados con entrada en catálogo en línea (1):

- Londres, British Library, c.63.f.25. (ejemplar único)

\section{Barcelona, Carles Amorós, 1531 (Bar1531): TCM}

Principales repertorios en línea: IB (65087), USTC (344232), BETA (manid 5907), COMEDIC (24)

Ejemplares localizados con entrada en catálogo en línea (1):

18.- El 28 de febrero de 2020 Iberian Books hacía público el descubrimiento de este nuevo ejemplar a través de su perfil de Twitter (https://twitter.com/IberianBooks/ status/1233426813596160001). Si bien la portada no se corresponde con ninguna de las conocidas, lo cierto es que aún habrá que comparar detalladamente este nuevo ejemplar con el de la British Library con signatura G.10223. para determinar si se trata de dos ediciones, dos emisiones o dos estados diferentes. Unos primeros resultados en Faulhaber (2020). 
- Viena, Österreichische Nationalbibliothek, 622185-B (ejemplar digitalizado en Austrian Books Online)

Ejemplares localizados sin entrada en catálogo en línea (2):

- Washington, The Folger Shakespeare Library, PO6426.A1 1531 (Rojas y antiguo autor 2000: $\mathrm{Bar}_{31}$; comprobado con la biblioteca)

- San Juan de Puerto Rico, La Casa del Libro (Rojas y antiguo autor 2000: $\mathrm{Bar}_{31}$, confirmado por el personal de la biblioteca)

24. Burgos, Juan de Junta, 12 de septiembre de 1531 (Bur1531): TCM

Principales repertorios en línea: IB (76884), USTC (348387), BETA (manid 5854), COMEDIC (25)

Ejemplares localizados con entrada en catálogo en línea (1):

- Barcelona, Biblioteca de Catalunya, Res 1522-12 (ejemplar único; digitalizado en Google Books y la Biblioteca Virtual Miguel de Cervantes)

\section{Venecia, Juan Bautista Pedrezano, 24 de octubre de 1531} (Ven1531): TCM

Principales repertorios en línea: IB (65086), USTC (344231 y 852868), BETA (manid 5867), COMEDIC (26)

Ejemplares localizados con entrada en catálogo en línea $(7)^{19}$ :

- Cambridge, Trinity College (University of Cambridge), G.26.45[2] (portada dañada)

- Londres, University of London, [E.P.] XPK [Calisto] SSR

- Madrid, Biblioteca Nacional de España, R/31236

- Madrid, Biblioteca Nacional de España, R/12435 (ejemplar digitalizado en la Biblioteca Digital Hispánica)

- Manchester, John Rylands Library (University of Manchester), R51723

- París, Bibliothèque Nationale de France, RES P-YG-4

- Venecia, Biblioteche Fondazione Giorgio Cini, FOAN TES 159 Ejemplares localizados sin entrada en catálogo en línea (1):

- Santander, Biblioteca Menéndez Pelayo, (463) (mútilo de la hoja $\mathrm{O} 4$, colección privada; $\mathrm{CCPB}$, se puede identificar con el ejemplar sin permiso de divulgación)

Ejemplares sin asignar, sin localizar o perdidos (5):

19.- El ejemplar de la Biblioteca de Catalunya con signatura Bon. 9-I-8 que consignan tanto BETA como IB y el USTC pertenece en realidad a la traducción italiana, [Venecia], Marchio Sessa, 10 de febrero de 1531. El USTC (852868) también atribuye erróneamente un ejemplar de la traducción italiana a esta edición: el de la British Library con signatura G.10159.. Asimismo, le atribuye un ejemplar de The Folger Shakespeare Library que pertenece en realidad a la edición Barcelona, Carles Amorós, 1531 (PQ6426.A1 1531). 
- Berlin, Staatsbibliothek Berlin, Xk 2691 (se envió con ocasión de la Segunda Guerra Mundial a Cracovia, Biblioteka Jagiellonska, donde lo localiza BETA, pero no aparece en el catálogo)

- Ejemplar Foulché Delbosc (BETA copid 8644)

- Ejemplar Huth (podría haber desaparecido durante el naufragio del Titanic)

- Ejemplar Gancia

- Ejemplar Maggs Bros

26. Medina del Campo, [Pedro de Tovans o Pedro de Castro], [ca. 1533-1537 o 1545] (Med1533-1545): TR

Principales repertorios en línea: IB (60152), USTC (341402), BETA (manid 5799), COMEDIC (37)

Ejemplares localizados con entrada en catálogo en línea (3):

- Londres, British Library, 243.a.8.

- Madrid, Biblioteca Nacional de España, R/3801

- Stuttgart, Württembergische Landesbibliothek Stuttgart, HB 421

27. Venecia, Stefano da Sabio, 10 de julio de 1534 (Ven1534): TCM

Principales repertorios en línea: IB (63965), USTC (343247), BETA (manid 5452), COMEDIC (27)

Ejemplares localizados con entrada en catálogo en línea (18):

- Barcelona, Biblioteca de Catalunya, Bon. 9-I-3 (ejemplar digitalizado en Google Books)

- Bérgamo, Biblioteca Civica Angelo Mai, CINQ.1.1464

- Boston, Boston Public Library, G.3356.12 (ejemplar digitalizado en Archive.org)

- Cambridge (EE.UU.), Houghton Library (Harvard University), *SC5 R6382C 1534

- Londres, British Library, G.10156.(1.).

- Madrid, Biblioteca Nacional de España, R/2877

- Madrid, Biblioteca Nacional de España, R/11599

- Madrid, Biblioteca Nacional de España, R/15030(1)

- Madrid, Biblioteca Nacional de España, CERV.SEDÓ/8647

- Milán, Biblioteca Nazionale Braidense, TT. 08. 0051/01 (portada deteriorada)

- Milwaukee ${ }^{20}$, University of Wisconsin-Milwaukee, PQ6426 .A1 1534

- Nueva York, Hispanic Society of America, PQ 6426 .A1 1534 (ejemplar digitalizado en TeXTRed) 
- París, Bibliothèque Nationale de France, RES-YG-302 (1)

- París, Bibliothèque Nationale de France, RESERVE 8-RE-6540 (ejemplar digitalizado en Gallica)

- Pesaro, Biblioteca Oliveriana, G - 15 - E - 14

- Udine, Seminario «P. Bertolla» del Seminario Arcivescovile ${ }^{21}$, 23129

- Venecia, Biblioteca del Museo Correr, OP.CICOGNA 0023 .7

- Viena, Österreschische Nationalbibliothek, 40.Mm.109 (ejemplar digitalizado en Austrian Books Online)

Ejemplares localizados sin entrada en catálogo en línea (6):

- Český Krumlov, Eggenberská knihovna [biblioteca del casti1lo], 25 D 5297 (colección privada; Archer et al. 2013: n 63)

- Bolonia, Biblioteca Comunale dell'Archiginnasio, BB.V.5 (Catálogo histórico Frati-Sorbelli, pero no está en EDIT16; confirmado por el personal de la biblioteca)

- Madrid, Biblioteca Francisco de Zabálburu, 37-49 (colección privada; CCPB)

- Roma, Biblioteca Angelica, O0.8.39/4 (EDIT16, encuadernado junto con la edición de la traducción italiana Venecia, Francesco di Alessandro Bindoni e Maffeo Pasini, junio de 1531)

- Santander, Biblioteca Menéndez Pelayo, I. 119 (colección privada; $\mathrm{CCPB}$, se puede identificar con el ejemplar sin permiso de divulgación)

- Turín, Biblioteca Real (EDIT16)

\section{Sevilla, Juan Cromberger, 1535 (Sev1535): TCM}

Principales repertorios en línea: IB (100443), USTC (351988), BETA (manid 5908), COMEDIC (28)

Ejemplares localizados sin entrada en catálogo en línea (1):

- Milán, Biblioteca Trivulziana, Triv.H.3579 (ejemplar único; Busquets 1998: $n^{\circ}$ 536)

29. Burgos, Juan de Junta, 28 de noviembre de 1536 (Bur1536): TCM

Principales repertorios en línea: IB (91749), USTC (351091), BETA (manid 5855), COMEDIC (30)

Ejemplares localizados con entrada en catálogo en línea (1):

- Barcelona, Biblioteca de Catalunya, Esp.65-8 (ejemplar único; digitalizado en Google Books) 
30. Sevilla, Domenico de Robertis, 12 de mayo de 1536 (Sev1536): TCM

Principales repertorios en línea: IB (92342), USTC (349922), BETA (manid 5856), COMEDIC (29)

Ejemplares localizados con entrada en catálogo en línea (1):

- Múnich, Bayerische Staatsbibliothek München, Res/4 P.o.hisp. 62 y (ejemplar digitalizado en MDZ Digitale Bibliothek y en Google Books) ${ }^{22}$

31. Toledo, Juan de Ayala, 28 de febrero de 1538 (Tol1538): TR

Principales repertorios en línea: IB (60153), USTC (341403), BETA (manid 5732), COMEDIC (31)

Ejemplares localizados con entrada en catálogo en línea (3):

- Génova, Biblioteca Universitaria di Genova, RARI R /5 /26

- Madrid, Biblioteca Nacional de España, R/4423

- Nueva York, Hispanic Society of America, PQ 6426 .A1 1538 (ejemplar digitalizado en TeXTRed)

Ejemplares sin asignar, sin localizar o perdidos (1):

- Roma, Biblioteca Nazionale Casanatense (aunque BETA, IB y el USTC consignan este ejemplar, el personal de la biblioteca me ha comunicado que no aparece).

32. Amberes, Guillaume du Mont, 28 de junio de 1539 (Amb1539): TCM

Principales repertorios en línea: IB (65089), USTC (440366), BETA (manid 5852), COMEDIC (32)

Ejemplares localizados con entrada en catálogo en línea (7):

- Aix-en Provence, Bibliothèque Méjanes, C.0928

- Cambridge, University of Cambridge, E.16.2

- Jena, Thüringer Universitäts- und Landesbibliothek Jena, 8 MS 25824 (ejemplar mútilo)

- Lawrence, Kenneth Spencer Research Library (University of Kansas), Summerfield A260

- Madrid, Real Academia de la Historia, 2/3941

- Nueva York, Hispanic Society of America, PQ 6426 .A1 1539

- París, Bibliothèque Mazarine, $8^{\circ} 22122$ [Res] (invertidos los cuadernillos i, k, l)

22.- Este ejemplar aparece en el USTC (347223) como única copia de una edición fantasma de Medina del Campo, s.n., 1536. El volumen comienza con la edición de Celestina que nos ocupa, pero continúa con una edición de la Tercera Celestina de Gaspar Gómez de Toledo, que es la que tiene dichos datos de impresión. En algún momento se perdió de vista que el ejemplar reúne dos obras diferentes y se le atribuyeron los datos de impresión de la segunda a la primera. 
Ejemplares localizados sin entrada en catálogo en línea (1):

- Boston, Public Libray, *D.178.24 (Whitney 1879: apéndice, 489)

Ejemplares sin asignar, sin localizar o perdidos (1):

- Ejemplar Quaritch-Paul (BETA copid 8488)

33. Salamanca, Pedro de Castro, 1540 (Sed): Sed

Principales repertorios en línea: IB (60150), USTC (341400), BETA (manid 5846), COMEDIC (34)

Ejemplares localizados con entrada en catálogo en línea (5):

- Madrid, Biblioteca Nacional de España, R/6601(1) (ejemplar expurgado y deteriorado)

- Madrid, Biblioteca Nacional de España, R/9683 (ejemplar mútilo de la portada y las dos últimas hojas; digitalizado en la Biblioteca Virtual Miguel de Cervantes)

- Nueva York, Hispanic Society of America, PQ 6433 .S445 S54 1540 (digitalizado en TeXTRed)

- Toledo, Biblioteca de Castilla-La Mancha, Res.22 (ejemplar mútilo; digitalizado en BIDICAM)

- Viena, Österreichische Nationalbibliothek, 59.D.35 (ejemplar digitalizado en Austrian Books Online)

34. Lisboa, Luis Rodríguez, 1540 (Lis1540): TCM

Principales repertorios en línea: IB (63966), USTC (343248), BETA (manid 5910), COMEDIC (33)

Ejemplares localizados con entrada en catálogo en línea (1):

- Londres, British Library, C.20.b.13. (ejemplar único)

35. Salamanca, Juan de Junta, 15 de marzo de 1543 (Sal1543): TR

Principales repertorios en línea: IB (60151), USTC (341401), BETA (manid 5865), COMEDIC (34)

Ejemplares localizados con entrada en catálogo en línea (1):

- Ripoll, Biblioteca Pública Lambert Mata, FONS MATA 671 (ejemplar único; digitalizado en ARGUS)

36. Sevilla, Herederos de Juan Cromberger, 12 de noviembre de 1543 (Sev1543): TCM

Principales repertorios en línea: IB (-), USTC (-), BETA (manid 6177), COMEDIC (36)

Ejemplares localizados con entrada en catálogo en línea (1):

- París, Bibliothèque Mazarine, $4^{\circ} 11070$ S [Res] (ejemplar único)

37. Amberes, Martín Nucio, [ca. 1544-1547] (Amb1544-1547): 


\section{TCM}

Principales repertorios en línea: IB (76088), USTC (440531), BETA (manid 5857 y 5853$)^{23}$, COMEDIC (41)

Ejemplares localizados con entrada en catálogo en línea (7):

- Augsburgo. Staats- und Stadtbibliothek Augsburg, LA 786

- Bruselas, Koninklijke Bibliotheek van België, L.P. 5.526 A

- Granada, Biblioteca del Hospital Real (Universidad de Granada), BHR/Caja IMP-4-050 (ejemplar digitalizado en DIGIBUG)

- Madrid, Biblioteca Nacional de España, R/40795

- Nueva York, Hispanic Society of America, PQ 6426 .A1 [1544]

- París, Bibliothèque Nationale de France ${ }^{24}$, 8-RE-6541

- Praga, Národní knihovna České republiky [Biblioteca Nacional de la República Checa] ${ }^{25}$, 9 L 000156/adl.1. (ejemplar digitalizado en Google Books)

Ejemplares sin asignar, sin localizar o perdidos (1):

- Colección privada sin especificar (IB y el USTC, pero lo incluimos en este apartado porque, al no estar identificado el propietario, es como si el ejemplar no estuviera localizado; podría ser el ejemplar que estuvo a la venta en la Librería Bardon que señala COMEDIC, puesto que ya no es posible encontrarlo en su espacio de IberLibro)

23.- Los dos registros de BETA no responden a una duplicación, sino que reflejan una interpretación diferente a la de los demás repertorios de las dos dataciones propuestas para esta edición por Penney (1954: 49-50), partidaria de 1544, y Peeters-Fontainas (1965: n²16), defensor de 1547. En lugar de considerar que los dos estudiosos están hablando de una misma edición, BETA crea un registro para 1544 (BETA manid 5853) y otro para 1547 (BETA manid 5857), alegando que «Es de suponer que se han cotejado los dos ejemplares [= BNE R/40795 y HSA PQ 6426.A1 [1544]] para cerciorarse de que se trata realmente de dos eds. diferentes». Aun así se señala la necesidad de confirmarlo definitivamente (también para el ejemplar de la Bibliothèque Royale de Belgique, con signatura L.P. 5.526 A), por lo que, mientras esto sucede, en lo que a este censo se refiere se tratará como una sola edición 1544-1547.

24.- COMEDIC atribuye el ejemplar de la Bibliothèque Nationale de France con signatura 8-RE-6541 a las ediciones de Amberes, Martín Nucio, 1545 y [1544-1547] (COMEDIC 38 y 41), sin decidirse por solo una de ellas. Sin embargo, la fecha entre corchetes en la entrada del catálogo digital hace suponer que el ejemplar no lleva el año de impresión en la portada, como sí hacen los ejemplares de la edición de 1545, por lo que puede descartarse que le pertenezca y asignarlo, con bastante seguridad, a la edición [1544-1547].

25.- BETA atribuye el ejemplar de la Biblioteca Nacional de la República Checa a Amberes, Martín Nucio, [1544] (BETA manid 5853), y a Amberes, Philippo Nucio, 1568 (BETA manid 6009), pero admite que «Probablemente corresponde a la ed. sin fecha de hacia 1544-1545». Esto viene corroborado por la ausencia de año de impresión en la portada del ejemplar checo (la edición de 1568 tiene el año en la portada). 


\section{Amberes, Martín Nucio, 1545 (Amb1545): TCM}

Principales repertorios en línea: IB (65090), USTC $(440367)^{26}, \mathrm{BE}-$ TA (manid 5858), COMEDIC (38)

Ejemplares localizados con entrada en catálogo en línea (2):

- Madrid, Biblioteca Nacional de España, R/3340 (ejemplar acéfalo y mútilo de D11, O11 y O12, con F5 y F6, F7 y F8, G5-6 y G7-8 intercambiadas) ${ }^{27}$

- Múnich, Bayerische Staatsbibliothek München, P.o.hisp 196 (ejemplar digitalizado en MDZ Digitale Bibliothek y Google Books)

- Nueva York, Hispanic Society of America, PQ 6426 .A1 1545a Ejemplares sin asignar, sin localizar o perdidos (1):

- Lovaina, Katholieke Universiteit (Peeters-Fontainas 1965: n 215; IB, el USTC y COMEDIC lo dan por existente sin pararse a considerar la anotación "avant 1940", que puede implicar que el ejemplar se perdió durante la Segunda Guerra Mundial, como muy amablemente me ha recordado el personal de la biblioteca de la Universidad Católica de Lovaina, donde el ejemplar no aparece)

\section{Zaragoza, Diego Hernández, 22 de abril de 1545 (Zar1545a): TCM}

Principales repertorios en línea: IB (63967), USTC (343249), BETA (manid 5851), COMEDIC (39)

Ejemplares localizados con entrada en catálogo en línea (3):

- Cambridge, University of Cambridge, Peterborough.H.2.7 (se trata de un ejemplar con las dos ediciones zaragozanas de 1545, aunque la entrada solo consigne la de Coci)

26.- IB y el USTC consignan varios ejemplares más que, sin embargo, están mal atribuidos a esta edición, ya que claramente pertenecen a Amb1544-1547, en cuanto que no llevan el año de impresión en portada. Se trata de los ejemplares de Augsburgo, Staats- und Stadtbibliothek, LA 786; París, Bibliothèque Nationale de France, 8-RE-6541, y Praga, Biblioteca Nacional de la República Checa, 9 L 000156/adl.1.. Respecto al ejemplar de Madrid, véase la apreciación que hacemos para el ejemplar de la Biblioteca Nacional de España con signatura R/3340.

27.- Al compararlo con el ejemplar con signatura R/40795 de la edición Amb1544-1547 comprobé que no pertenecen a la misma edición y procedí a cotejarlo con la digitalización del ejemplar con signatura P.o.hisp 196 de la Bayerische Staatsbibliothek de la edición de Amb1545, confirmando que se trata de un ejemplar de esta última. Esto resulta especialmente evidente en $\mathrm{A} 3 \mathrm{r}$, donde el título aparece con una distribución diferente del texto y con la primera línea en mayúsculas (diferencia recurrente también el el título en A4v) y no se deja una línea en blanco antes del primer «Prosigue», como en el ejemplar alemán. También se puede comprobar en A6, que en Amb1545 tiene 33 líneas en el recto y 31 en el verso, mientras que en Amb1544-1547 tiene 32 en cada cara, o en la capital xilográfica de A9v (diferente en las dos ediciones), los errores de signatura (por ejemplo, E3 aparece por D3 en Amb1545, así como cae la signatura de la sexta hoja en los cuadernillos A, B y C) y, por supuesto, los reclamos. 
- Londres, British Library, C.63.e.18.

- Madrid, Biblioteca Histórica Municipal de Madrid, Par 224 (ejemplar digitalizado en Memoria de Madrid)

Ejemplares localizados sin entrada en catálogo en línea (1):

- Boston, Boston Public Library, G.3356.13 (Whitney 1879: 69)

40. Zaragoza, Jorge Coci para Pedro Bernuz y Bartolomé de Nágera, 17 de junio de 1545 (Zar1545b): TCM

Principales repertorios en línea: IB (65091), USTC (344235), BETA (manid 5847), COMEDIC (40)

Ejemplares localizados con entrada en catálogo en línea (4):

- Cambridge, University of Cambridge, Peterborough.H.2.7 (se trata de un ejemplar con las dos ediciones zaragozanas de 1545, aunque la entrada solo consigne la de Coci)

- Madrid, Biblioteca Nacional de España, R/39668

- Nueva York, Hispanic Society of America, PQ 6426 .A1 1545b (ejemplar digitalizado en TeXTRed)

- Wolfenbüttel, Herzog August Bibliothek, A: 556.1 Quod. (1) (ejemplar con portada deteriorada)

\section{Sevilla, Jácome Cromberger, 1550 (Sev1550): TCM}

Principales repertorios en línea: IB (65092), USTC (344236), BETA (manid 5911), COMEDIC (42)

Ejemplares localizados con entrada en catálogo en línea (2):

- Módena. Biblioteca Estense, ALPHA P 010015

- Viena, Österreichische Nationalbibliothek, 622184-B (ejemplar digitalizado en Austrian Books Online)

Ejemplares sin asignar, sin localizar o perdidos (1):

- Göttingen, Niedersächsische Staats- und Universitätsbibliothek Göttingen, 8 FAB II, 266 (perdido)

42. Venecia, Gabriel Giolito de Ferrariis y sus hermanos, 20 de enero de 1553 (Ven1553): TCM

Principales repertorios en línea: IB (63968), USTC (343250), BETA (manid 5864), COMEDIC (44)

Ejemplares localizados con entrada en catálogo en línea (18)를

28.- El ejemplar de la Biblioteca civica Angelo Mai de Bérgamo que consignan IB y el USTC para esta edición con casi total seguridad es una errata por el ejemplar con signatura CINO.1.1232, de la edición Venecia, Gabriel Giolito de Ferrariis y hermanos, 1553 de la Cecaria. Para los ejemplares de la Università degli Studi di Padua y de la Biblioteca Menéndez Pelayo recogidos en IB y en el USTC (y en BETA y en COMEDIC, en el caso del último ejemplar), véanse las notas 28 y 30. 
- Ausgburgo, Staats- und Stadtbibliothek Augsburg, LA 785 (ejemplar digitalizado en MDZ Digitale Bibliothek y en Google Books)

- Bolonia, Reale Collegio di Espagna, C X 6

- Burnaby, W.A.C. Bennett Library (Simon Fraser University), PQ 6427.5 C35

- Cambridge, University of Cambridge, F155.d.2.7

- Cuenca, Seminario Mayor o Conciliar de San Julián, CU-BSC, 209-D-04

- Londres, British Library, 243.b.38.

- Los Angeles, Charles E. Young Research Library (University of California, Los Angeles), Z233.G4 R64ce 1553

- Madrid, Biblioteca Nacional de España, R/3240

- Madrid, Biblioteca Nacional de España, R/10178

- Madrid, Biblioteca Nacional de España, CERV.SEDÓ/8639 (ejemplar incompleto y manipulado)

- Montpellier, Bibliothèque universitaire historique de Médecine (Université de Montpellier), Ac 387 in-16 p1

- Nueva York, Hispanic Society of America, PQ 6426 .A1 1553

- Roma, Biblioteca Nazionale Centrale, 69. 6.D.16 (ejemplar digitalizado en Google Books)

- Santiago de Compostela, Universidade de Santiago de Compostela, 2216 (ejemplar incompleto falto de portada, digitalizado en MINERVA)

- Siena, Biblioteca Comunale degli Intronati, VI/2 O 89 [1]

- Siena, Biblioteca Comunale degli Intronati, VI/2 O 89 [2]

- Turín. Biblioteca Nazionale Universitaria de Torino, F X.191

- Utrecht. Universiteit Utrecht, MAG: ODA 9083

Ejemplares localizados sin entrada en catálogo en línea (2):

- Toledo, Cigarral del Carmen (colección particular; COMEDIC)

- Zaragoza, Biblioteca de Alfonso Fernández González (colección particular; COMEDIC)

Ejemplares sin asignar, sin localizar o perdidos (8):

- Ejemplar Foulché-Delbosc (BETA copid 8663)

- Ejemplar Morante (BETA copid 8660)

- Ejemplar Morante (BETA copid 8661)

- Ejemplar Peeters-Fontainas (BETA copid 8665)

- Ejemplar Potier (BETA copid 8658)

- Ejemplar Salvá-Heredia (BETA copid 8664)

- Ejemplar Seillière (BETA copid 8662)

- Ejemplar Soleinne (BETA copid 8659) 


\section{Zaragoza, Pedro Bernuz, 1554 (Zar1554): TCM}

Principales repertorios en línea: IB (-), USTC (-), BETA (manid 6178), COMEDIC (45)

Ejemplares sin asignar, sin localizar o perdidos (1):

- Colección particular, poseedor sin revelar (ejemplar único, colección privada; Rojas 2018)

\section{Zaragoza, Agustín Millán, 1555 (Zar1555): TCM}

Principales repertorios en línea: IB (65094), USTC (344237), BETA (manid 5940), COMEDIC (46)

Ejemplares localizados con entrada en catálogo en línea (1):

- Nueva York, Hispanic Society of America, PQ 6426 .A1 1555 (ejemplar único)

45. Venecia, Gabriel Giolito de Ferrariis y sus hermanos, 1556 [20 de enero de 1553] (Ven1553-1556): TCM

Principales repertorios en línea: IB (65095), USTC (344238), BETA (manid 5941), COMEDIC (43)

Ejemplares localizados con entrada en catálogo en línea (11) 29 :

- Breslavia, Uniwersytet Wrocławski (Universidad de Breslavia), 451566

- Gotha, Universitäts- und Forschungsbibliothek Erfurt/Gotha, Phil 8o 01499/01 (02)

- Madrid, Biblioteca de Palacio Real, I/B/206

- Múnich, Bayerische Staatsbibliothek München, P.o.hisp. 196 k (ejemplar digitalizado en MDZ Digitale Bibliothek y Google Books)

- Nueva York, Hispanic Society of America, PQ 6426 .A1 1556

- Padua, Biblioteca Universitaria di Padova ${ }^{30}$, B.105.b.233.1 (falta la marca del impresor y el colofón)

- Princeton, Princeton University, 3176.68.324

- San Petersburgo, Росси́йская национа́льная библиоте́ка [Biblioteca Nacional de Rusia], 6.20.7.26

- Schwäbisch Hall, Ratsbibliothek (Stadtarchiv Schwäbisch Hall), Oct.294

- Venecia, Biblioteca del Museo Correr, OP.CICOGNA 0090 .12

29.- EDIT16 consigna un ejemplar en la Biblioteca civica Angelo Mai de Bérgamo, que IB y el USTC asignan a la edición Ven1553, pero en ambos casos se trata de una errata por la edición Venecia, Gabriel Giolito de Ferrariis y sus hermanos, 1553 de la Cecaria, con signatura CINQ.1.1232 (ver nota 27).

30.- Este ejemplar aparece erróneamente atribuido a Ven1553 en IB y el USTC, pero la entrada catalográfica de la Universidad de Padua no deja lugar a confusión y asigna muy claramente este ejemplar a Ven1553-1556. 
- Wolfenbüttel, Herzog August Bibliothek, A: 107.37 Eth Ejemplares localizados sin entrada en catálogo en línea (2):

- Bolonia, Biblioteca Comunale dell'Archiginnasio, AA.V.35 (Catálogo histórico Frati-Sorbelli, pero no está en EDIT16; confirmado por el personal de la biblioteca)

- Santander, Biblioteca Menéndez Pelayo ${ }^{31}$, (445) (colección privada; $\mathrm{CCPB}$, se puede identificar con el ejemplar sin permiso de divulgación)

46. Estella, Adrián de Amberes, 24 de enero de 1557 (Est1557): $\operatorname{Tr}$

Principales repertorios en línea: IB (65096), USTC (344239), BETA (manid 6010), COMEDIC (47)

Ejemplares localizados con entrada en catálogo en línea (1):

- Múnich, Bayerische Staatsbibliothek München, Res/P.o.hisp. 197 (ejemplar único; digitalizado en MDZ Digitale Bibliothek y en Google Books)

47. Amberes, viuda de Martín Nucio, 1558 (Amb1558): TCM

Principales repertorios en línea: IB (63969), USTC (440195), BETA (manid 6011), COMEDIC (48)

Ejemplares localizados con entrada en catálogo en línea (4):

- Hannover, Gottfried Wilhelm Leibniz Bibliothek, Lr 4591

- Londres, British Library, C.125.cc.3.

- París, Bibliothèque Nationale de France, 8-BL-16057

- Rennes, Les Champs Libres, 84771

Ejemplares localizados sin entrada en catálogo en línea (1):

- Parma, Biblioteca Palatina di Parma, Sal.O.XVI.41596 (COMEDIC, pero también en el catálogo histórico digitalizado)

48. Estella, Adrián de Amberes, 3 de diciembre de 1560 (Est1560): TR

Principales repertorios en línea: IB (106158), USTC (352668), BETA (manid 5839), COMEDIC (49)

Ejemplares localizados sin entrada en catálogo en línea (1):

- Cracovia, Biblioteka Jagiellonska, Cim. o. 1282 (ejemplar único; Griffin 1980: 567-569)

31.- COMEDIC duplica este ejemplar para las ediciones Venecia, Gabriel Giolito de Ferrariis y sus hermanos, 20 de enero de 1553, y Venecia, Gabriel Giolito de Ferrariis y sus hermanos, 1556 [20 de enero de 1553] (COMEDIC 43 y 44) que, además, BETA, IB y el USTC asignan a Ven1553. Sin embargo, de acuerdo con la descripción de Ediciones de teatro español en la Biblioteca de Menéndez Pelayo (Vega García-Luengos et al. 2012: n. 787 ) no cabe duda de que pertenece a Ven1553-1556. 


\section{Barcelona, Claudi Bornat, 1561 (Bar1561): TCM}

Principales repertorios en línea: IB (65097), USTC (344240), BETA (manid 6013), COMEDIC (50)

Ejemplares localizados con entrada en catálogo en línea (1):

- Nueva York, Hispanic Society of America, PQ 6426 .A1 1561 (ejemplar único)

\section{Cuenca, Juan de Cánova, 1561 (Cue1561): TCM}

Principales repertorios en línea: IB (76886), USTC (348388), BETA (manid 5840), COMEDIC (52)

Ejemplares localizados sin entrada en catálogo en línea (1):

- Cracovia, Biblioteka Jagiellonska, Cim. 5689 (ejemplar único; Griffin 1980: 567-569)

51. Valladolid, Francisco Fernández de Córdoba, 7 de enero de 1562 [1561] (Vall1561-1562): TCM

Principales repertorios en línea: IB (60158), USTC (341407), BETA (manid 6012), COMEDIC (51)

Ejemplares localizados con entrada en catálogo en línea (1):

- Madrid, Biblioteca Nacional de España, R/30464 (ejemplar único) ${ }^{32}$

52. Sevilla, Sebastián Trujillo, 1562 (Sev1562): TCM

Principales repertorios en línea: IB (65098), USTC (344241), BETA (manid 6014), COMEDIC (53)

Ejemplares localizados con entrada en catálogo en línea (1):

- Nueva York, Hispanic Society of America, PQ 6426 .A1 1562 (ejemplar único)

\section{Alcalá de Henares, Francisco de Cormellas y Pedro de Robles} [para Juan de Orta], 1563 (Alc1563): TCM

Principales repertorios en línea: IB (60168), USTC (341417), BETA (manid 6004), COMEDIC (54)

Ejemplares localizados con entrada en catálogo en línea (2):

- Berkeley, The Bancroft Library (University of California, Berkeley), t PQ6426.A1 1563

32.- Con COMEDIC, considero que el ejemplar de la Universidad de Oviedo consignado por IB y el USTC es un error. No solo el ejemplar no aparece en ningún otro repertorio, sino que la supuesta signatura que se le atribuye no se parece a las de la biblioteca de la Universidad de Oviedo. La única vez que una signatura similar, R/33.224, aparece asignada a uno de libros de su fondo, lo hace asociada a la Historia de el abbad don Juan de Montemayor (Valladolid, Francisco Fernández de Córdoba, 1562), hoy con signatura CEA-205, en la Historia de la imprenta en Valladolid (siglos XVI-XVII) (Marsá Vilá 2007: n. ${ }^{3}$ 364). Obviamente, la equivocación deriva de la coincidencia en el lugar, el año y el impresor de las dos ediciones. 
- Madrid, Biblioteca Nacional de España, R/8360

54. Medina del Campo, Francisco del Canto, 1563 (Med1563): TCM

Principales repertorios en línea: IB (106159), USTC (352669), BETA (manid 5841), COMEDIC (55)

Ejemplares localizados sin entrada en catálogo en línea (1):

- Cracovia, Biblioteka Jagiellonska, Cim. o.790 (ejemplar único; Griffin 1980: 572-573)

55. Medina del Campo, Francisco del Canto, 1566 (Med1566): TCM

Principales repertorios en línea: IB (98523), USTC (5046473), BETA (manid 6032), COMEDIC (56)

Ejemplares localizados con entrada en catálogo en línea (1):

- Madrid, Biblioteca de Palacio Real, I.C.266 (ejemplar único)

56. Barcelona, Claudi Bornat, 1566 (Bar1566): TCM

Principales repertorios en línea: IB (65099), USTC (344242), BETA (manid 5455), COMEDIC (57)

Ejemplares localizados con entrada en catálogo en línea (3):

- Ann Arbor, University of Michigan, PQ 6426 .A1 1566 (mútilo de la hoja B1)

- Roma, Biblioteca Nazionale Centrale, 6. 16.G.41

- Fermo, Biblioteca Civica Romolo Spezioli, 1 b 1357

Ejemplares localizados sin entrada en catálogo en línea (1):

- Toledo, Biblioteca del Cigarral del Carmen (colección particular; COMEDIC)

\section{Amberes, Philippo Nucio, 1568 (Amb1568): TCM}

Principales repertorios en línea: IB (63970), USTC (440196) ${ }^{33}$, BETA (manid 6009), COMEDIC (58)

Ejemplares localizados con entrada en catálogo en línea (13)

- Bruselas, Koninklijke Bibliotheek van België, L.P. 5.569 A

- Hannover, Gottfried Wilhelm Leibniz Bibliothek, Lr 4592

- Londres, British Libray, 1163.a.42. (ejemplar digitalizado en Google Books)

33.- La edicion Amberes, Philippo Nucio, 1563 del USTC (440319) es, casi sin lugar a dudas, una errata por esta de 1568. Asimismo, el USTC (44019) consigna un ejemplar con signatura 9 L 156/adl.1. 920 (pero, en realidad, 9 L 000156/adl.1.) en la Biblioteca Nacional de la República Checa que no pertenece a esta edición, sino a Amb1544-1547.

34.- Los dos ejemplares de la Houghton Library de la Universidad de Harvard que consigna COMEDIC no han podido ser confirmados por el personal de la biblioteca, por lo que debe tratarse de un error. 
- Madison, Memorial Library (University of Wisconsin-Madison), 715363

- Madrid, Biblioteca Nacional de España ${ }^{35}, \mathrm{R} / 7836$

- Madrid, Real Academia de la Historia, 1/2726

- Mannheim, Schloss Schneckenhof (Universität Mannheim), Mf s 052

- Milán, Biblioteca Sormani, VET.C VET.58

- Múnich, Bayerische Staatsbibliothek München, P.o.hisp. 198 (ejemplar digitalizado en MDZ Digitale Bibliothek y Google Books)

- Múnich, Ludwig-Maximilians-Universität München, 0001/8 P.hisp. 55

- París, Bibliothèque Mazarine, 8045278 [Res] (mútilo del último cuadernillo; las hojas 5 y 6 están mal encuadernadas tras las hojas 7 y 8)

- Solothurn, Zentralbibliothek Solothurn, ZBS QC 184

- Urbino, Università degli Studi di Urbino, 001 D-09 001

Ejemplares sin asignar, sin localizar o perdidos (2):

- ¿Lovaina?, ¿Biblioteca de Peeters-Fontainas? (colección privada; Peeters-Fontainas 1965: n²18; pero COMEDIC no tiene en cuenta que la biblioteca de Peeters-Fontainas se dispersó en 1978 con la venta de sus ejemplares por Sotheby's, y aunque es probable que se trate del ejemplar al que IB y el USTC hacen referencia como "en colección privada", no sirve de nada si no está identificado el propietario)

- Lovaina, Katholieke Universiteit (Peeters-Fontainas 1965: $n^{\circ} 218$; IB, el USTC y COMEDIC lo dan por existente sin pararse a considerar la anotación "avant 1940", que puede implicar que el ejemplar se perdió durante la Segunda Guerra Mundial, como muy amablemente me ha recordado el personal de la biblioteca de la Universidad Católica de Lovaina, donde el ejemplar no aparece)

\section{Madrid, Pierres Cosin [para Antón García], 1569 (Mad1569): TCM}

Principales repertorios en línea: IB (65100), USTC (344243), BETA (manid 5848), COMEDIC (59)

Ejemplares localizados con entrada en catálogo en línea (1):

- Roma, Biblioteca Nazionale Centrale Vittorio Emanuele II, 35.10.A.23 (ejemplar digitalizado en Google Books)

Ejemplares localizados sin entrada en catálogo en línea (1): 
- Nueva York, New York University ${ }^{36}$, PQ 6426 .A1 1569 (ejemplar digitalizado en TeXTRed)

59. Salamanca, Mathias Mares, 1569 (Sal1569): TCM

Principales repertorios en línea: IB (65101), USTC (344244), BETA (manid 6179), COMEDIC (60)

Ejemplares localizados con entrada en catálogo en línea (1):

- Nueva York, Hispanic Society of America, PQ 6426 .A1 1569

60. Sevilla, Alonso de la Barrera, 1569 (Sev1569): TCM

Principales repertorios en línea: IB (76887), USTC (348389), BETA (manid 6029), COMEDIC (61)

Ejemplares localizados con entrada en catálogo en línea (1):

- Nantes, Bibliothèque Municipale de Nantes, 30256R (ejemplar único $)^{37}$

\section{Alcalá de Henares, Juan de Villanueva [para Pedro del Bosque], 1569 (Alc1569): TCM}

Principales repertorios en línea: IB (60147), USTC (341398), BETA (manid 6015), COMEDIC (62)

Ejemplares localizados con entrada en catálogo en línea (1):

- Madrid, Biblioteca Nacional de España, R/31686 (ejemplar único; falto de la hoja B5 y con marcas de censura y expurgo)

\section{Salamanca, Mathias Gast [para Simón Borgoñón], 1570 (Sal1570):} TCM

Principales repertorios en línea: IB (60159), USTC (341408), BETA (manid 5850), COMEDIC (63)

Ejemplares localizados con entrada en catálogo en línea (9):

- Buenos Aires, Biblioteca Nacional Mariano Moreno, 6 FD

- Florencia, Biblioteca Nazionale Centrale, MAGL.3.8.452./b

- Livorno, Biblioteca Labronica Francesco Domenico Guerrazzi, BASTOGI 094-V- 0082

- Londres, British Library, 11725.a.8. (ejemplar digitalizado en Google Books)

- Madrid, Biblioteca Nacional de España, R/7110 (falta la fe de erratas)

36.- COMEDIC ubica erróneamente este ejemplar en la Hispanic Society of America, probablemente debido a que el facsímil digital de este ejemplar aparece con la marca de agua de la misma, pero lo que la Hispanic Society ha hecho ha sido adquirir los derechos de reproducción, únicamente.

37.- BETA, IB y el USTC aún no han incorporado este ejemplar y siguen marcando esta edición como sin ejemplares conocidos. 
- Madrid, Biblioteca Nacional de España, R/31914 (ejemplar deteriorado)

- Nueva York, Hispanic Society of America, PQ 6426 .A1 1570 (ejemplar digitalizado en TeXTRed)

- Oviedo, Universidad de Oviedo, CEA-399

- París, Bibliothèque Mazarine, $8^{\circ} 21966$ B-2 [Res]

Ejemplares localizados sin entrada en catálogo en línea (2):

- Abbeville, Bibliothèque Municipale d'Abbeville, FA 16 E 500 (COMEDIC, confirmado con la propia biblioteca)

- Boston, Boston Public Library, G.3356.14 (Whitney 1879: 69-70) Ejemplares sin asignar, sin localizar o perdidos (1)

- Ejemplar de Techener-Morante (BETA copid 8692)

63. Alcalá de Henares, Juan de Villanueva, 1570-1571 (Alc1570): TCM

Principales repertorios en línea: IB (-), USTC (-), BETA (manid 6180), COMEDIC (64)

Ejemplares localizados con entrada en catálogo en línea (1):

- Toledo, Biblioteca de Castilla La Mancha, 1-919 (medio pliego en la encuadernación)

\section{Toledo, Francisco de Guzmán, 1573 (Tol1573): TCM}

Principales repertorios en línea: IB (60160), USTC (341409), BETA (manid 6017), COMEDIC (65)

Ejemplares localizados con entrada en catálogo en línea (3):

- Madrid, Biblioteca Nacional de España, R/1449

- Madrid, Biblioteca Nacional de España, R/8281 (ejemplar mútilo de las hojas A1 y A2)

- París, Bibliothèque Mazarine, $8^{\circ} 44271$ [Res]

Ejemplares sin asignar, sin localizar o perdidos (1):

- ¿Segovia?. ¿Catedral? (COMEDIC consigna este ejemplar, pero el personal del archivo no lo ha localizado ni entre los fondos actuales ni en los registros)

65. Salamanca, Pedro Lasso Vaca, 1573 (Sal1573): TCM

Principales repertorios en línea: IB (-), USTC (-), BETA (manid 6181), COMEDIC (66)

Ejemplares localizados con entrada en catálogo en línea (1):

- Voralberg, Voralberger Landesbibliothek, Emsiana-1573/1 (ejemplar único, en proceso de restauración o reencuadernación)

38.- No figura el ejemplar Foulché-Delbosc (BETA copid 8651) por ser, con total seguridad, el ejemplar de la Biblioteca Nacional Mariano Moreno con signatura 6 FD (cf. Olivetto 1998: no 2). 
66. Alcalá de Henares, Juan de Lequerica para Juan Gutiérrez, 1575 (Alc1575): TCM

Principales repertorios en línea: IB (65102), USTC (344245), BETA (manid 6018), COMEDIC (67)

Ejemplares localizados con entrada en catálogo en línea (2):

- Barcelona, Universitat de Barcelona, 07 B-51/8/27 (anotaciones de expurgo y censura)

- Chicago, University of Chicago, PQ6426.A1 1575

67. Salamanca, Álvaro Ursino, 1575 (Sal1575): TCM

Principales repertorios en línea: IB (65103), USTC (344246), BETA (manid 5844), COMEDIC (68)

Ejemplares localizados con entrada en catálogo en línea (2):

- Nueva York, Hispanic Society of America, PQ 6426 .A1 1575a

- Oxford, Bodleian Library (University of Oxford), Vet. G1 f.18

68. Sevilla, Alonso Picardo para Pedro Miguel, 1575 (Sev1575): TCM

Principales repertorios en línea: IB (65104), USTC (344247), BETA (manid 6019), COMEDIC (69)

Ejemplares localizados con entrada en catálogo en línea (2):

- Nueva York, Hispanic Society of America, PQ 6426.A1 1575b

- Viena, Österreichisches Nationalbibliothek, 59.L.48 (ejemplar digitalizado en Austrian Online Books)

69. Valencia, Juan Navarro, 1575 (Val1575): TCM

Principales repertorios en línea: IB (60161), USTC (341410), BETA (manid 6016), COMEDIC (70)

Ejemplares localizados con entrada en catálogo en línea (3) ${ }^{39}$ :

- Aix-en Provence, Bibliothèque Méjanes, C. 7955

- Madrid, Biblioteca Nacional de España, R/7870

- Madrid, Biblioteca Nacional de España, R/11094

\section{Salamanca, Pedro Lasso, 1577 (Sal1577): TCM}

Principales repertorios en línea: IB (65105), USTC (344248), BETA (manid 6020), COMEDIC (71)

Ejemplares localizados con entrada en catálogo en línea (1):

- Roma, Biblioteca Casanatense, VOL MISC.2441 1 (ejemplar único; digitalizado en Google Books) 
71. Sevilla, Alonso de la Barrera para Luis Torrero, 1582 (Sev1582): TCM

Principales repertorios en línea: IB (60163), USTC (341412), BETA (manid 6021), COMEDIC (72)

Ejemplares localizados con entrada en catálogo en línea (1):

- Madrid, Biblioteca Nacional de España, R/24843 (ejemplar único)

72. Medina del Campo, Francisco del Canto para Claudio Curlet y Pablo Ascanio, 1582 (Med1582): TCM

Principales repertorios en línea: IB (60162), USTC (341411), BETA (manid 5845), COMEDIC (73)

Ejemplares localizados con entrada en catálogo en línea (1):

- Madrid, Biblioteca Nacional de España, R/7491 (ejemplar único; digitalizado en la Biblioteca Digital Hispánica)

73. Barcelona, Hubert Gotard, 1585 (Bar1585): TCM

Principales repertorios en línea: IB (65106), USTC (344249), BETA (manid 5900), COMEDIC (74)

Ejemplares localizados con entrada en catálogo en línea (1):

- Viena, Österreichische Nationalbibliothek, *38.M.189 (ejemplar único)

Ejemplares sin asignar, sin localizar o perdidos (1):

- Ejemplar Paul (BETA copid 8712)

74. Alcalá de Henares, Juan Gracián para Juan Boyer, 1586 (Alc1586): TCM

Principales repertorios en línea: IB (60164), USTC (341413), BETA (manid 5451), COMEDIC (75)

Ejemplares localizados con entrada en catálogo en línea (2):

- Madrid, Biblioteca Nacional de España, R/7045 (ejemplar censurado)

- París, Bibliothèque Nationale de France, 8-BL-16058

Ejemplares localizados sin entrada en catálogo en línea:

- Boston, Boston Public Library, XD.170B.8 (Whitney 1879: 70)

75. Salamanca, Juan y Andrés Renaut para Claudio Curlet, 1590 (Sal1590): TCM

Principales repertorios en línea: IB (60165), USTC (341414), BETA (manid 5861), COMEDIC (76)

Ejemplares localizados con entrada en catálogo en línea (6):

- Cambridge, University of Cambridge, F159.c.8.2 
- Copenhague, Det Kongelige Bibliotek, 177:1, 230

- Madrid, Biblioteca Nacional de España, U/4005 (ejemplar digitalizado en la Biblioteca Digital Hispánica)

- Nueva York, Hispanic Society of America, PQ 6426 .A1 1591 (mútilo de las hojas 5, 254 y todas las que siguen a la 260; portada y hojas 2 y 114-120 dañadas)

- París, Bibliothèque Mazarine, $8^{\circ} 45385$ [Res] (mútilo de la hoja 145)

- Ratisbona, Staatliche Bibliothek Regensburg40,999/Hisp.13 (ejemplar digitalizado en la MDZ Digitale Bibliothek y en Google Books)

Ejemplares localizados sin entrada en catálogo en línea (1):

- Santander, Biblioteca Menéndez Pelayo, (120) (colección particular; CCPB, se puede identificar con el ejemplar sin permiso de divulgación)

Ejemplares sin asignar, sin localizar o perdidos (1):

- Ejemplar Foulché Delbosc (BETA copid 8655)

\section{Alcalá de Henares, Hernán Ramírez, 1591 (Alc1591): TCM}

Principales repertorios en línea: IB (60166), USTC (341415), BETA (manid 5899), COMEDIC (77)

Ejemplares localizados con entrada en catálogo en línea (1):

- Madrid, Biblioteca Nacional de España, R/10197 (ejemplar único, con marcas de censura y deteriorado)

Ejemplares localizados sin entrada en catálogo en línea (1):

- Bristol, Bristol Central Library (IB y USTC; confirmado por el personal de la biblioteca)

\section{7. [Leiden], Oficina Plantiniana, 1595 (Plant1595): TCM}

Principales repertorios en línea: IB (63971), USTC (440197 y 440368), BETA (manid 5453), COMEDIC (79)

Ejemplares localizados con entrada en catálogo en línea (40) ${ }^{41}$ :

- Aix-en Provence. Bibliothèque Méjanes, C. 3905

- Amberes. Museum Plantin-Moretus, A 1092

40.-BETA, COMEDIC, IB y el USTC atribuyen este ejemplar a la Bayerische Staatsbibliothek de Múnich, pero se debe a que el catálogo de esta recoge la digitalización.

41.- BETA y el USTC incluyen un ejemplar del Clare College de Cambridge del que no he encontrado noticia en ningún otro lugar, por lo que debe de tratarse de algún cruce con el ejemplar de la Universidad de Cambridge o de un ejemplar fantasma. Igualmente, BETA, IB y el USTC atribuyen a esta edición un ejemplar de la Bibliothèque Royale de Belgique que debe de resultar de un cruce con alguno de los dos ejemplares de la edición [Leiden], Oficina Plantiniana, 1599, con las signaturas VB 6.930 A RP y VB 6.464 30v A 4 RP, respectivamente. Lo más probable es que se trate de este último, ya que no aparece recogido en ninguno de los tres repertorios como ejemplar de Plant1599. 
- Bilbao, Biblioteca Foral de Bizkaia, R-43 (1) (ejemplar digitalizado en Lau Haizeetara Biltegia)

- Boston, Boston Public Library, XD.170B.7

- Cambridge. University of Cambridge, F159.e.6.3

- Ciudad del Vaticano, Biblioteca Apostolica Vaticana, Stamp. De.Luca.VI. 2405

- Copenhague, Det Kongelige Bibliotek, 177:1, 230

- Detmold, Lippische Landesbibliothek Theologische Bibliothek Detmold, 02-F 378

- Durham, Routh Library (University of Durham), Routh. 70.F. 24

- Göttingen, Niedersächsische Staats- und Universitätsbibliothek Göttingen, 8 FAB II, 270 RARA

- Grenoble, Bibliothèque Municipale de Grenoble, F.1834

- Jena, Thüringer Universitäts und Landesbibliothek Jena, 8 MS 18709

- Lawrence, Kenneth Spencer Research Library (University of Kansas), Summerfield B2592

- Leiden, Universiteitsbibliotheek Leiden, 20643 G 13

- Londres, British Library, 1072.f.1.

- Londres, British Library, 1072.b.33

- Londres, British Library, G.10157

- Madison, Memorial Library (University of Wisconsin-Madison), 903055 noncurrent

- Madrid, Biblioteca Nacional de España, CERV.SEDÓ/8645

- Madrid, Biblioteca Nacional de España, R/9174

- Madrid, Biblioteca Nacional de España, R/12957

- Madrid, Biblioteca Nacional de España, R/23665

- Madrid, Biblioteca de Palacio Real, I/B/205

- Madrid, Museo Lázaro Galdiano, R 7-7-16

- Nueva York, Hispanic Society of America, PQ 6426 .A1 1595a

- Oviedo, Universidad de Oviedo, CEA-128

- Oxford, Bodleian Library (University of Oxford), $8^{\circ} \mathrm{C} 45$ Art.

- Oxford, All Souls College (University of Oxford), nn.15.13

- Oxford, Wadham Colelge (University of Oxford), g 16.17

- París, Bibliothèque Nationale de France, RES-YG-305

- París, Bibliothèque Nationale de France, YG-2657 (mútilo de la portada y las hojas 1-6)

- París, Bibliothèque Mazarine, $8^{\circ} 22123$

- Parma, Biblioteca Palatina, CC XI.27245

- Ripoll, Biblioteca Lambert Mata, FONS MATA 700 (ejemplar digitalizado en Pandora)

- San Marino (EE.UU.), Huntington Library, 82607 
- San Petersburgo, Росси́йская национа́льная библиоте́ка [Biblioteca Nacional de Rusia], 6.20.8.65

- Troyes, Médiathèque Jacques Chirac, aa.16.3653

- Utrecht, Universiteitsbibliotheek Utrecht, MAG : Z oct 67 (Rariora)

- Viena, Österreichische Nationalbibliothek, 76.J.3 ALT PRUNK

- Williamstown, Williams College, sin signatura

Ejemplares localizados sin entrada en catálogo en línea (3):

- Carcassonne, Médiathèque Intercommunale de Carcassone, GEN X 003312 (CCFr)

- Valencia, Biblioteca municipal Serrano Morales, 2/242 (CCPB)

- Zaragoza, Biblioteca de Alfonso Fernández González (colección privada; COMEDIC, es posible que se corresponda con el ejemplar en colección privada consignado por IB y el USTC)

Ejemplares sin asignar, sin localizar o perdidos (6):

- Berlín, Staatsbibliothek Berlin, Xk 2699 (ejemplar perdido durante la Segunda Guerra Mundial)

- Ejemplar Foulché-Delbosc (BETA copid 8656)

- Ejemplar Jonghe (BETA copid 8669)

- Ejemplar Morante-Seillière-Brooke (BETA copid 8666)

- Ejemplar Crofts (BETA copid 8667)

- Ejemplar Soleinne-Salvá-Heredia (BETA copid 8657)

\section{Tarragona, Felipe Roberto, 1595 (Tarr1595): TCM}

Principales repertorios en línea: IB (60167), USTC (341416), BETA (manid 5454), COMEDIC (80)

Ejemplares localizados con entrada en catálogo en línea (6):

- Cambridge (EE.UU.), Houghton Library (Harvard University), *SC5 R6382C 1595

- Ciudad del Vaticano, Biblioteca Apostolica Vaticana, Stamp. Barb.KKK.I.54

- Madrid, Biblioteca Nacional de España, R/8776

- Madrid, Fundación Lázaro Galdiano, R 7- 6-13

- Múnich, Bayerische Staatsbibliothek München, P.o.hisp. 199 (ejemplar digitalizado en MDZ Digitale Bibliothek y Google Books)

- Nueva York, Hispanic Society of America, PQ 6426 .A1 1595a

Ejemplares localizados sin entrada en catálogo en línea (1):

- Zaragoza, Biblioteca particular de Alfonso Fernández González (colección privada; COMEDIC)

Ejemplares sin asignar, sin localizar o perdidos (2):

- Ejemplar Font de Robinat (BETA copid 8670)

- Ejemplar Rosell y Rubert (BETA copid 8671) 


\section{Sevilla, Fernando de Lara, 1596 (Sev1596): TCM}

Principales repertorios en línea: IB (65108), USTC (344251), BETA (manid 6005), COMEDIC (81)

Ejemplares localizados con entrada en catálogo en línea (3):

- Estrasburgo, Bibliothèque nationale et universitaire de Strasbourg, CD.122.735

- Madrid, Bibllioteca Nacional de España ${ }^{42}$, CERV.SEDÓ/8646

- Nueva York, Hispanic Society of America, PQ 6426 .A1 1596

Ejemplares localizados sin entrada en catálogo en línea (1):

- Zaragoza, Biblioteca particular de Alfonso Fernández González (colección privada; COMEDIC)

Ejemplares sin asignar, sin localizar o perdidos (4):

- Berlín, Staatsbibliothek Berlin, Xk 2700 (perdido durante la Segunda Guerra Mundial)

- Ejemplar Balagué Llibreria Antiquària (ya no figura en su espacio de IberLibro y no me consta que el nuevo poseedor esté identificado)

- Ejemplar Debure Frères (BETA copid 8688)

- Ejemplar Foulché-Delbos (BETA copid 8686)

\section{0. [Leiden], Oficina Plantiniana, 1599 (Plant1599): TCM}

Principales repertorios en línea: IB (63972), USTC (440198, 441617 y 452507), BETA (manid 5882), COMEDIC (82)

Ejemplares localizados con entrada en catálogo en línea (81):

- Aberystwyth, Llyfrgell Genedlaethol Cymru [Biblioteca Nacional de Gales], b99 L4 (3)

- Albi, Mediathèque Pierre-Amalric, RES. ROCH. 05888

- Amberes, Erfgoedbibliotheek Hendrik Conscience, C 79100

- Amberes, Museo Plantin-Moretus, A 904

- Angers, Bibliothèque Municipale d'Angers, BL 2453 - format 1

- Barcelona, Biblioteca de Catalunya, Bon. 9-I-2 (digitalizado en Google Books)

- Barcelona, Biblioteca de Catalunya, Res 522-12º (digitalizado en Google Books)

- Beaune, Bibliothèque Municipale Gaspard Monge, Ancien A 712

- Berna, Universität Bern, MUE Bong VI 190

- Bonn, Universitäts- und Landesbibliothek Bonn, Fd 352

- Breslavia, Uniwersytet Wrocławski (Universidad de Breslavia), 451607

- Breslavia, Uniwersytet Wrocławski (Universidad de Breslavia), 451608 
- Bruselas, Koninklijke Bibliotheek België, VB 6.930 A RP

- Bruselas, Koninklijke Bibliotheek België43, VB 6.464 30v A 4 RP

- Cambridge. Trinity College (University of Cambridge), G.26.36[1]

- Cambridge. University of Cambridge, Y.12.46[sel.e]

- Cambridge. University of Cambridge, Hisp.8.59.3

- Chantilly, Bibliothèque du Château de Chantilly, XI-F-099

- Chicago, Newberry Library, Case Y 722 .R616

- Ciudad del Vaticano, Biblioteca Apostolica Vaticana, Stamp. Chig.VI.264

- Coutances, Médiathèque Communautaire de Coutances, 3458

- Copenhague, Det Kongelige Bibliotek, 177:1, 230

- Cremona, Biblioteca Statale di Cremona, FA.40.2.5

- Dijon, Bibliothèque Municipale de Dijon, 7887

- Dijon, Bibliothèque Municipale de Dijon, Belloguet I-245

- Dillingen, Studienbibliothek Dillingen-Donau, Mag/XVII 501

- Edimburgo, National Library of Scotland, [Ag].8/1.5

- Edimburgo, University of Edinburgh, De. 5.106

- Erfurt, Universitätsbibliothek Erfurt, 03 - Lp. $8^{\circ} 00551$

- Filadelfia, Kislak Center (University of Pennsylvania), 868 R63C 1599

- Göttingen, Niedersächsische Staats- und Universitätsbibliothek Göttingen, 8 FAB II, 272 RARA

- Halle/Saale, Universitäts- und Landesbibliothek Sachsen-Anhalt, Dk 1224

- Halle/Saale, Universitäts-und Landesbibliothek Sachsen-Anhalt, an Dl 2593 (3) (1)

- Hamburgo, Staats-und Universitätsbibliothek Hamburg, A/16863

- Leiden, Universiteit Leiden, 20643 G 23

- Leiden, Universiteit Leiden, 20643 G 24

- Londres, British Library, 11726.a.19.

- Londres, London Library, Small Books

- Madrid, Biblioteca Nacional de España, CERV.SEDÓ/8644

- Madrid, Biblioteca Nacional de España, R/2006

- Madrid, Biblioteca Nacional de España, R/1507

- Madrid, Biblioteca Nacional de España, R/8406

- Madrid, Biblioteca Nacional de España, R/13410

- Madrid, Biblioteca Nacional de España, U/8527 (ejemplar digitalizado en la Biblioteca Digital Hispánica) 
- Madrid, Biblioteca Histórica Municipal, I/123 (ejemplar digitalizado en Memoria de Madrid)

- Madrid, Biblioteca Histórica Marqués de Valdecilla (Universidad Complutense), BH FG 1765 (ejemplar digitalizado en Hathi Trust y Google Books)

- Madrid, Museo Lázaro Galdiano, R 8- 8-26

- Madrid, Real Academia de la Historia, 14/7293

- Manchester, John Rylands Library (University of Manchester), Special Collections 14747

- Metz, Médiathèque Verlaine, Fonds patrimoniaux Réserve patrimoniale Niveau 3 Q 1325

- Moscú, Российская государственная библиотека [Biblioteca Estatal de Rusia], 50-6459047

- Múnich, Ludwig-Maximilians-Universität München, 0001/8 P.hisp. 11

- Nancy, Bibliothèque Stanislas, Res. 10242a

- Nueva York, Hispanic Society of America, PQ 6426 .A1 1599a c. 1

- Nueva York, Hispanic Society of America, PQ 6426 .A1 1599a c. 2

- Oldenburg, Landesbibliothek Oldenburg, SPR XVII A 57,1

- Oviedo, Universidad de Oviedo, CEA-024

- Oviedo, Universidad de Oviedo, CEA-012

- Oxford, St Hugh's College (University of Oxford), RBS GG17 (ROJ)

- Oxford, Taylor Institution (University of Oxford), VET. SPAN.I.A.23

- Oxford, Wadham College (University of Oxford), g30.4

- París, Bibliothèque Nationale de France, 8-BL-16059

- París, Bibliothèque Nationale de France, 8-BL-16060

- París, Bibliothèque Nationale de France, THN-115

- París, Bibliothèque Nationale de France, YG-2656

- París, Bibliothèque Nationale de France, 8-RE-6542

- París, Bibliothèque Sainte-Geniève, 8 Y SUP 1130 RES

- París, Bibliothèque Mazarine, $8^{\circ} 22130$ B

- París, Bibliothèque de la Sorbonne, VCM 6= 14270

- Poitiers, Médiathèque François-Mitterrand, D 3707

- Roma, Biblioteca Vallicelliana, S.BOR Q.III 222

- Rouen, Bibliothèque Municipale Villon, Mt p 7321

- Salzburgo, Paris Lodron Universität Salzburg, I 73403

- Schwerin, Landesbibliothek Mecklenburg-Vorpommern Günther Uecker, OI V 890

- Stuttgart, Württembergische Landesbibliothek Stuttgart, HB 420

- Trento, Biblioteca Diocesana Vigilianum, dvd-S.M.C. 89 
- Toulouse, Bibliothèque d'Étude et Patrimonie de Toulouse, Res. D XVI 291

- Ventimiglia, Biblioteca Civica Aprosiana, CINO D 528

- Vicenza, Biblioteca Civica Bertoliana, D 005 2bis 031 MAGAZZINO

- Viena, Österreichische Nationalbibliothek, *38. M. 100 (ejemplar digitalizado en Austrian Books Online)

- Wolfenbüttel, Herzog August Bibliothek, A: 137.15 Eth

Ejemplares localizados sin entrada en catálogo en línea (4):

- Madrid, Biblioteca Francisco Zabálburu, BFZ: 37-50 (CCPB)

- Santander, Biblioteca Menéndez Pelayo (CCPB)

- Winchester, Winchester College, M12 Bk1936 (LDH)

- Zaragoza, Biblioteca particular de Alfonso Fernández González (COMEDIC; podría ser el ejemplar que IB y el USTC consignan como en colección privada)

Ejemplares sin asignar, sin localizar o perdidos (14):

- ¿Bérgamo?, ¿Biblioteca Civica Angelo Mai? (COMEDIC consigna este ejemplar, pero el personal de la biblioteca me ha confirmado no lo tienen)

- Berlín, Staastbibliothek, Xk 2704 (perdido durante la Segunda Guerra Mundial)

- Ejemplar Escobet y Cots (BETA copid 8698)

- Ejemplar Glanville-Sunderland-Quaritch (BETA copid 8713)

- Ejemplares Luzarche-Quaritch-Paul (BETA copid 8711)

- Ejemplar Marqués de la Romana (BETA copid 8716)

- Ejemplar Morante-Peeters-Fontainas (BETA copid 8691)

- Ejemplar Salvá-Heredia-Boix (BETA copid 8695)

- Ejemplar Seillière-Chivers (BETA copid 8714)

- Ejemplar Seillière-Ellis (BETA copid 8704)

- Ejemplar Techener-Foulché-Delbosc (BETA copid 8693)

- Ejemplar Turner (BETA copid 8715)

- ¿Lovaina?, ¿Biblioteca particular de Peeters Fontainas? (colección privada; Peeters-Fontainas 1965: n 220; COMEDIC no tiene en cuenta que la biblioteca de Peeters Fontainas fue subastada en 1978 en Sotheby's y, por lo tanto, dispersada, y aunque podría ser el ejemplar que IB y el USTC consignan como en colección privada, al no estar identificado el poseedor es como si no estuviera localizado)

- Weimar, Herzogin Anna Amalia Bibliothek, $12^{\circ}$ XL : 43 (ejemplar perdido durante el incendio de la biblioteca en 2004) 
81. Madrid, Andrés Sánchez para Miguel Martínez, 1601 (Mad1601): TCM

Principales repertorios en línea: IB (B73488), USTC (5026501) BETA (manid 5627), COMEDIC (N/A)

Ejemplares localizados con entrada en catálogo en línea (14):

- Abbeville, Bibliothèque Municipale d'Abbeville, FA-17-E-32

- Chicago, University of Chicago, PQ6426.A1 1601

- Cincinnati, KLAU Library (Hebrew Union College), Freidus E R

- Hanover, Dartmouth Library, PQ6426 .A1 1601

- Madrid, Biblioteca Nacional de España, R/3750 (hojas total o parcialmente manuscritas; ejemplar digitalizado en la Biblioteca Digital Hispánica)

- Moscú, Российская государственная библиотека [Biblioteca Estatal de Rusia], IV-исп. $8^{\circ}$

- Múnich, Bayerische Staatsbibliothek München, P.o.hisp. 200 (ejemplar digitalizado en MDZ Digitale Bibliotehk y Google Books)

- Nueva York, Hispanic Society of America, PQ 6426 .A1 1601 c. 1

- Nueva York, Hispanic Society of America, PQ 6426 .A1 1601 c. 2

- Oviedo, Universidad de Oviedo, CEA-418

- París, Bibliothèque Nationale de France, 8-RE-6543

- París, Bibliothèque Nationale de France, RES 8-Z ADLER-454

- Ponta Delgada, Biblioteca Publica e Arquivo Distrital, JC/A AR.2 A/157 RES

- Wolfenbüttel, Herzog August Bibliothek, A: 130.17 Eth

Ejemplares localizados sinn entrada en catálogo en línea (2):

- Colorno, Biblioteca Comunale Pier Luigi Belloni (IB y USTC, confirmado con la biblioteca)

- Praga, Národni Knihovna České Republiky [Biblioteca Nacional de la República Checa], II Hh 5 (Kašpar 1983: n 63)

Ejemplares sin asignar, sin localizar o perdidos (4):

- Berlín, Staatsbibliothek, 8" Xk 2705 (perdido durante la Segunda Guerra Mundial)

- Tres ejemplares en colecciones privadas subastados en 1978 (IB y USTC)

82. Zaragoza, Carlos de Lavayen y Juan Larumbre, 1607 (Zar1607): TCM

Principales repertorios en línea: IB (B7890), USTC (5030302), BETA (manid 6182), COMEDIC (N/A)

Ejemplares localizados con entrada en catálogo en línea (9): 
- Besançon, Bibliothèque Municipale de Besançon, 243105

- Madrid, Biblioteca Nacional de España, R/11954

- Madrid, Biblioteca del Palacio Real, I/C/269

- Nueva York, Hispanic Society of America, PQ 6426.A1 1607

- Oxford, Worcester College (University of Oxford), ZZ.4.4

- París, Bibliothèque Nationale de France ${ }^{45}$, 8-RE-6544

- París, Bibliothèque Nationale de France, 8-BL-16061

- Santiago de Compostela, Universidade de Santiago de Compostela, 16641 (ejemplar digitalizado en MINERVA)

- Viena, Österreichische Nationalbibliothek, *38.L.96 ALT PRUNK (ejemplar digitalizado en Austrian Books Online)

Ejemplares localizados sin entrada en catálogo en línea (1):

- Lincoln, Catedral de Lincoln (IB y USTC; confirmado por el personal de la biblioteca)

Ejemplares sin asignar, sin localizar o perdidos (1):

- Berlín, Staatsbibliothek Berlin, Xk 2707 (perdido durante la Segunda Guerra Mundial)

\section{Madrid, Juan de la Cuesta, 1619 (Mad1619): TCM}

Principales repertorios en línea: IB (B72910), USTC (5026182), BETA (manid 6183), COMEDIC (N/A)

Ejemplares localizados con entrada en catálogo en línea (9):

- Bilbao, Biblioteca Foral de Bizkaia, R-884 (ejemplar digitalizado en Lau Haizeetara Biltegia)

- Ciudad del Vaticano, Biblioteca Apostolica Vaticana, Stamp. Ross.6907

- Glasgow, University of Glasgow, Sp Coll Bi7-1.19

- Madrid, Biblioteca Nacional de España, R/11216

- Madrid, Biblioteca Nacional de España, R/30479

- Moscú, Российская государственная библиотека [Biblioteca Estatal de Rusia], IV-исп. $8^{\circ}$

- Nueva York, Hispanic Society of America, PQ 6426 .A1 1619

- París, Bibliothèque Nationale de France, YG-2654

- Oviedo, Universidad de Oviedo, CGII-0386

Ejemplares localizados sin entrada en catálogo en línea (3):

- Évora, Biblioteca Pública de Évora, S. N. XXIX/1 (IB y USCT, pero no aparece en PORBASE y la biblioteca no ha respondido)

- Lisboa, Academia das Ciências, BACL 1180132 (IB y USTC, pero no aparece en PORBASE; el personal de la biblioteca ha confirmado su presencia) 
- Santander, Biblioteca de Menéndez Pelayo, (722) (Vega García-Luengos et al. 2012: n. ${ }^{\circ}$ 789)

\section{Milán, Juan Baptista Bidelo, 1622 (Mil1622): TCM}

Principales repertorios en línea: IB (B80470), USTC (5031529), BETA (manid 6184), COMEDIC (N/A)

Ejemplares localizados con entrada en catálogo en línea (9):

- Bonn, Universität Bonn, Fd 352/9

- Detroit, Purdy-Kresge Library (Wayne State University), PQ6426. A1 1622

- Ithaca, Cornell University, PQ6426.A1 1622 tiny

- Múnich, Bayerische Staatsbibliothek München, P.o.hisp. 200 e (ejemplar digitalizado en MDZ Digital Bibliothek y Google Books)

- Múnich, Bayerische Staatsbibliothek München, P.o.hisp. 200 d (ejemplar digitalizado en MDZ Digital Bibliothek y Google Books)

- Múnich, Bayerische Staatsbibliothek München, Rem.IV 277 (ejemplar digitalizado en MDZ Digitale Bibliothek)

- Nueva York, Hispanic Society of America, PQ 6426 .A1 1622

- París, Bibliothèque Sainte-Geniève, 8 Y 2955 (2) INV 5522 RES (P.1)

- Syracuse, Bird Library (Syracuse University), 863.23 R74

Ejemplares localizados sin entrada en catálogo en línea (1):

- Bolonia, Biblioteca Comunale dell'Archiginnasio, 2/BB.II.57 (IB y USTC; pero en el catálogo histórico Frati-Sorbelli, la ficha correspondiente a este ejemplar lleva la signatura BB.V.38)

Ejemplares sin asignar, sin localizar o perdidos (2):

- Berlín, Staatsbibliothek Berlin, Xk 2710 (perdido durante la Segunda Guerra Mundial)

- ¿Londres?, ¿British Library? (Rojas y antiguo autor 2000: Mil622; pero el personal de la biblioteca no ha encontrado ningún rastro de dicho ejemplar)

85. Madrid, viuda de Alonso Martín, 1631 [1632] (Mad1631-1632): Exp

Principales repertorios en línea: IB (B70894 y B28243), USTC (5024793 y 5010801), BETA (manid 5424), COMEDIC (N/A)

Ejemplares localizados con entrada en catálogo en línea (14):

- Augsburgo, Staats-und Stadtbibliothek Augsburg, LA 788 (ejemplar digitalizado en MDZ Digitale Bibliothek y Google Books) 
- Barcelona, Biblioteca de Catalunya, Res.2308-12o (mútilo de las hojas 60 y 146; ejemplar digitalizado en Google Books)

- Cagliari, Biblioteca Universitaria di Cagliari, A 7111

- Copenhague, Det Kongelige Bibliothek, 177:1, 230

- Londres, British Library, 1072.c.19.

- Madrid, Biblioteca Nacional de España, CERV.SEDÓ/8648 (mútilo de la última hoja)

- Madrid, Biblioteca Nacional de España, R/12948

- Madrid, Biblioteca Nacional de España, U/1607 (hojas 127 y 134 restauradas con el texto a mano; ejemplar digitalizado en la Biblioteca Digital Hispánica)

- Madrid, Real Academia Española, 38-XI-43

- Nueva York, Hispanic Society of America, PQ 6426 .A1 1632 (ejemplar digitalizado en TeXTRed)

- Oviedo, Universidad de Oviedo, CEA-445

- París, Bibliothèque Nationale de France, YG-2655

- París, Bibliothèque Sainte-Geneniève, 8 Y 2955 INV 5521 RES

- Parma, Biblioteca Palatina, CC IX.27379

Ejemplares localizados sin entrada en catálogo en línea (1):

- Tudanca, Casona de Tudanca, 1-2-14 (CCPB)

Ejemplares sin asignar, sin localizar o perdidos (3):

- Ejemplar Kraus (BETA copid 3425)

- Ejemplar Vindel (BETA copid 3431)

- ¿Madrid?, ¿Ateneo de Madrid? (BETA; aunque el ejemplar aparecía hasta hace poco en el CCPB - ya no figura - el personal de la biblioteca no lo ha hallado, por lo que debe haber habido algún error)

86. Pamplona, Carlos Labayén, 1633 (Pam1633): Bil

Principales repertorios en línea: IB (B82765), USTC (5033246), BETA (manid 6185), COMEDIC (N/A)

Ejemplares localizados con entrada en catálogo en línea (8) ${ }^{46}$ :

- Barcelona, Biblioteca de Cataluña, Res. 1518-12

- Londres, British Library, 11726.aa.21.

- Madrid, Biblioteca Nacional de España, R/12390 (ejemplar deteriorado)

- Orléans, Médiathèque Municipale d'Orléans, Rés.D2214

- París, Bibliothèque Nationale de France, 8-RE-6545

- París, Bibliothèque de la Sorbonne, VCM 6=12484

- Valladolid, Biblioteca Histórica de Santa Cruz, U/Bc BU 04117

- Viena, Österreichische Nationalbibliothek, 69.M.50 (ejemplar digitalizado en Austrian Books Online) 


\section{Ruan, Charles Osmont, 1633 (Rua1633): Bil}

Principales repertorios en línea: IB (-), USTC (6813936) ${ }^{47}$, BETA (manid 6186), COMEDIC (N/A)

Ejemplares localizados con entrada en catálogo en línea (4):

- Londres, British Library, 1072.e.28.

- Nueva York, Hispanic Society of America, PQ 6426.A1 1633

- Stuttgart, Württembergische Landesbibliothek Stuttgart, HB 2785;

- Toulouse, Bibliothèque Municipale de Toulouse, Res D XVII-343 (ejemplar digitalizado en Tolosana)

\section{Ruan, Charles Osmont, 1634 [1633] (Rua1633-1634): Bil}

Principales repertorios en línea: IB (B75550), USTC (6813067 y 5027901) ${ }^{48}$, BETA (manid 6187), COMEDIC (N/A)

Ejemplares localizados con entrada en catálogo en línea (14):

- Aix-en-Provence, Bibliothèque Méjanes, D. 0267

- Edimburgo, National Library of Scotland, G.22.g.23

- Madrid, Biblioteca Nacional de España, R/241

- Madrid, Biblioteca Nacional de España, CERV.SEDÓ/8640 (mútilo de la portada en español; la portada en francés es lo único que queda del cuadernillo inicial sin signatura)

- Múnich, Bayerische Staatsbibliothek München, P.o.gall. 646 (ejemplar digitalizado en MDZ Digitale Bibliothek y Google Books)

- Nueva York, Hispanic Society of America, PQ 6426 .A1 1634

- Oxford, All Souls College (University of Oxford), nn.15.12

- París, Bibliothèque Nationale de France ${ }^{49}$, GD-743

- París, Bibliothèque Nationale de France, 8-BL-16054

- París, Bibliothèque Nationale de France, 8-BL-16055

- París, Bibliothèque Nationale de France, Y2-6343

- París, Bibliothèque Nationale de France, RES-YG-308 (ejemplar mútilo de las portadas, por lo que podría pertenecer a otra edición)

- París, Bibliothèque Nationale de France, RES-YF-3666

- Valencia, Biblioteca Histórica (Universitat de València), T/1168 Ejemplares localizados sin entrada en catálogo en línea (3):

47.- El ejemplar único de la Biblioteca Nacional de Escocia que el USTC consigna para esta edición parece ser el que, con signatura G.22.g.23, asigno a Rua1633-1634.

48.- De los ejemplares que consigna el USTC (6813067), solo podemos confirmar el de Aixen-Provence y el de Oxford. Sospecho que el ejemplar de la British Library es una errata por el de la edición Rua1633-1634 o el de la edición Rua1644, mientras que el de Toulouse probablemente sea una errata por el ejemplar tolosano de Rua1633-1634, por lo que no los consigno.

49.- La lista de Lobera et al. (Rojas y antiguo autor 2000: Rua634) asigna este ejemplar a Rua1644, pero el catálogo de la Biblioteca Nacional de Francia lo atribuye a Rua1633-1634. 
- Boston, Boston Public Library, D.170b.3 (Whitney 1879: 70)

- Boston, Boston Public Library, D.170b.4 (ejemplar mútilo de la portada francesa, por lo que no es posible saber si se trata de esta edición o de la anterior sin examinarlo antes; Whitney 1879: 70)

- Ejemplar en venta en TodoColección (artesianox)

Ejemplares sin asignar, sin localizar o perdidos (2):

- Colección privada, ejemplar vendido por Sotheby's en 1978 (IB y USTC)

- Colección privada, ejemplar vendido por Cristies en 2013 (IB y USTC)

89. Ruan, Charles Osmont, 1644 (Rua1644): Bil

Principales repertorios en línea: IB (-), USTC (6812310), BETA (manid 6188), COMEDIC (N/A)

Ejemplares localizados con entrada en catálogo en línea (2):

- Londres, British Library, 242.h.30.

- París, Bibliothèque Nationale de France, 8-RE-6546 


\section{Obras citadas}

Álvarez Márouez, María del Carmen (2007), La impresión y el comercio de libros en la Sevilla del quinientos, Sevilla, Universidad de Sevilla.

- (2009), Impresores, libreros y mercaderes de libros en la Sevilla del quinientos, Zaragoza, Pórtico.

ArCHER, Robert et al. (2013), Bohemia hispánica: fondos españoles de los siglos XV a XVII en bibliotecas checas, Barcelona, Reial Academia de Bònes Lletres.

BETA, «Bibliografía Española de Textos Antiguos», base de datos bibliográfica. Accedido el 01 de noviembre de 2020, disponible on-line $<$ https://bancroft.berkeley.edu/philobiblon/beta_en.html>.

BotTA, Patrizia (2001), "Edizione critica de La Celestina di Fernando de Rojas (dall'Atto VIII alla fine)", edición digital. Accedido el 01 de noviembre de 2020, disponible on-line $<$ http://rmcisadu.let.uniroma1.it/ celestina/celest.htm>.

BotTA, Patrizia, y Víctor Infantes (1999), «Nuevas bibliográficas de la Tragicomedia de Calisto y Melibea (Zaragoza, Jorge Coci, 1507)", Revista de Literatura Medieval, 11, pp. 179-208.

Busauets, Loreto (1998), Impresos españoles del siglo XVI en Lombardía, Kassel, Reichenberger.

BVB, «Bibliotheksverbund Bayern», catálogo colectivo. Accedido el 01 de noviembre de 2020, disponible on-line <http://www.bib-bvb.de>.

Castillejo Benavente, Arcadio (2019), La imprenta en Sevilla en el siglo XVI (1521-1600), ed. Cipriano López Lorenzo, Sevilla - Córdoba, Editorial Universidad de Sevilla - UCOPress.

CCFr, "Catalogue Collectif de France», catálogo colectivo. Accedido el 01 de noviembre de 2020, disponible on-line <https://ccfr.bnf.fr>.

CCPB, «Catálogo Colectivo de Patrimonio Bibliográfico Español», catálogo colectivo. Accedido el 01 de noviembre de 2020, disponible on-line $<$ http://catalogos.mecd.es/CCPB/cgi-ccpb/abnetopac>.

CERL Thesaurus, "Thesaurus. Consortium of European Research Libraries (CERL)", tesauro bibliográfica. Accedido el 01 de noviembre de 2020, disponible on-line <https://thesaurus.cerl.org>.

COMEDIC, "Catálogo de obras medievales impresas en castellano", base de datos bibliográfica, ficha, La Celestina. Accedido el 01 de noviembre de 2020, disponible on-line <https://comedic.unizar.es/index/ $\mathrm{read} / \mathrm{id} / 322>$.

Cornejo, Rafael (1977), "Bibliografía de La Celestina», en "La Celestina» y su contorno social: Actas del I Congreso Internacional sobre "La Celestina", ed. Manuel Criado de Val, Madrid, Hispam, pp. 552-584.

Escudero y Perosso, Francisco (1894), Tipografía hispanlese. Anales bibliográficos de la ciudad de Sevilla desde el establecimiento de la imprenta hasta fines del siglo XVIII, Madrid, Sucesores de Rivadeneyra. 
FAUlHaber, Charles B. (2015), «Philobiblon 2015 n. 4 (Septiembre): la Biblioteca Lobkowicz y la edición de Celestina de 1523 (Posdata 2018-02-01)», UC Berkeley Library Update, entrada de blog, 02 de septiembre de 2015. Accedido el 01 de noviembre de 2020, disponible on-line <https://update.lib.berkeley.edu/2015/09/02/philobiblon-2015-n-4-setiembre-labiblioteca-lobkowicz-y-la-edicion-de-celestina-de-1523/>.

- (2020), "Una nueva emisión de la edición de Sevilla: Cromberger, 1525, de Celestina», UC Berkeley Library Update, entrada de blog, 07 de marzo de 2020. Accedido el 01 de noviembre de 2020, disponible online <https://update.lib.berkeley.edu/2020/03/07/una-nueva-emisonde-celestina-sevilla-cromberger-1525>.

Fernández Valladares, Mercedes (2019), "Otra enigmática Tragicomedia de Calisto y Melibea con la data contrahecha de 1502: análisis tipográfico y ensayo de ecdótica iconográfica (con una nueva Cárcel de amor (1520)", en Libros, lecturas y reescrituras, ed. María Jesús Lacarra, Zaragoza, Universidad de Zaragoza, pp. 463-502.

GBV, "Gemeinsamer Bibliotheksverbund», catálogo colectivo. Accedido el 01 de noviembre de 2020, disponible on-line <http://www.gbv.de $>$.

Givanel Mas, Juan (1919), "Contribución al estudio bibliográfico de la Celestina y descripción de un rarísimo ejemplar de dicha obra", Revista Crítica Hispano-Americana, 5.3-4, pp. 77-121.

Griffin, Clive (1980), "Four Rare Editions of La Celestina», Modern Language Review, 75.3, pp. 561. DOI: https://doi.org/10.2307/3727974

- (2013), "More Books from the Sixteenth Century Printed in Seville by the Cromberger Dynasty», Bulletin of Spanish Studies, 90.4-5, pp. 679-718. DOI: 10.1080/14753820.2013.802588.

GW, "Gesamtkatalog der Wiegendrucke», base de datos bibliográfica. Accedido el 01 de noviembre de 2020, disponible on-line <https://www. gesamtkatalogderwiegendrucke.de>.

HBZ, «Nordrhein-Westfälischer Bibliotheksverbund (Hochschulbibliothekszentrum des Landes Nordrhein-Westfalen)", catálogo colectivo. Accedido el 01 de noviembre de 2020, disponible on-line $<$ https://www.hbz-nrw.de>.

HeBIS, "Hessisches Bibliotheksinformationssystem», catálogo colectivo. Accedido el 01 de noviembre de 2020, disponible on-line <https:// www.hebis.de>.

IB, «Iberian Books», base de datos bibliográfica. Accedido el 01 de noviembre de 2020, disponible on-line <https://iberian.ucd.ie>.

ICCU SBN, "Catalogo del Servizio Bibliotecario Nazionale», catálogo colectivo. Accedido el 01 de noviembre de 2020, disponible on-line $<$ http://www.sbn.it/opacsbn/opac/iccu/free.jsp >.

INFANTES, Víctor (2007), «El laberinto cronológico y editorial de las primitivas impresiones de Celestina (1497-1514). Con una marginalia bibliographica al cabo", en Actas del Simposio Internacional 1502-2002: Five 
Hundred Years of Fernando de Rojas" "Tragicomedia de Calisto y Melibea", ed. Juan Carlos Conde, Nueva York, Hispanic Seminary of Medieval Studies, pp. 3-87.

ISTC, «Incunabula Short Title Catalogue», base de datos bibliográfica. Accedido el 01 de noviembre de 2020, disponible on-line <http:// www.bl.uk/catalogues/istc>.

Kašpar, Oldřich (1983), Soupis španělských tiskủ bývalé zámecké knihovny v Roudnici nad Labem, nyni deponovaných ve Státní knihovně ČSR v Praze [Registro de los impresos españoles de la antigua biblioteca del Castillo de Roudnice nad Labem actualmente depositada en la Biblioteca Estatal de la República Socialista Checa en Praga], Praha, Státní knihovna ČSR.

KOPV, «Kooperativer Bibliotheksverbund Berlin-Brandenburg», catálogo colectivo. Accedido el 01 de noviembre de 2020, disponible on-line $<$ https://www.kobv.de>.

Krapf, Eugenio (1900a), "Ediciones de La Celestina. Ensayo de un catálogo cronológico de las mismas», en La Celestina: Tragicomedia de Calisto y Melibea, ed. Marcelino Menéndez y Pelayo, Vigo, E. Krapf, pp. III-LIX.

- (1900b), "Una reseña de las principales traducciones de la Celestina», en La Celestina: Tragicomedia de Calisto y Melibea, ed. Marcelino Menéndez y Pelayo, Vigo, E. Krapf, pp. LX-XCIX.

KVK, «Karlsruher Virtueller Katalog», metabuscador de catálogos bibliográficos. Accedido el 01 de noviembre de 2020, disponible on-line $<$ https://kvk.bibliothek.kit.edu>.

Lacarra, María Jesús (2019), «La Tragicomedia de Calisto y Melibea en Zaragoza (1507-1607): los modelos iconográficos y su pervivencia», en La fisonomía del libro medieval y moderno entre la funcionalidad, la estética y la información, dir. Manuel José Pedraza Gracia, coord. Camino Sánchez Oliveira y Alberto Gamarra Gonzalo, Zaragoza, Universidad de Zaragoza, pp. 237-250.

LHD, "Library Hub Discover», catálogo colectivo. Accedido el 01 de noviembre de 2020, disponible on-line <https://discover.libraryhub.jisc. ac.uk>.

Marsá Vilá, María (2007), Materiales para una historia de la imprenta en Valladolid (siglos XVI y XVII), León, Universidad de León.

Martín Abad, Julián (2017), "La Tragicomedia de Calisto y Melibea de "Sevilla, 1502": Una nueva edición (BETA manid 5905)», UC Berkeley Library Update, entrada de blog, 22 de abril de 2017. Accedido el 01 de noviembre de 2020, disponible on-line <https://update.lib.berkeley.edu/2017/04/22/la-tragicomedia-de-calisto-y-melibea-de-sevilla1502-una-nueva-edicion>.

OlivetTo, Georgina (1998), «Ejemplares de Celestina de la colección Foulché-Delbosc en la Biblioteca Nacional de la República Argentina», Celestinesca, 22.1, pp. 67-74. 
Palau y Dulcet, Antonio (1950), Manual del librero hispano-americano. Tomo III "C-Comyn", Barcelona, Librería anticuaria de A. Palau.

PaolinI, Devid (2010), "Ediciones de La Celestina anteriores al siglo XIX en la Biblioteca Nacional de España", Revista de Literatura Medieval, 22, pp. 351-359.

- (2013), «Sobre una edición (des)conocida de La Celestina y viejos errores bibliográficos», Revista de Literatura Medieval. 25, pp. 303-305.

Peeters-Fontainas, Jean F. (1965), Bibliographie des impressions espagnoles des Pays-Bas méridionaux, Nieuwkoop, De Graaf.

Penney, Clara (1954), The Book Called "Celestina" in the Library of the Hispanic Society of America, New York, Hispanic Society of America.

PORBASE, «Base Nacional de Dados Bibliográficos», catálogo colectivo. Accedido el 01 de noviembre de 2020, disponible on-line <http://porbase.bnportugal.pt>.

RojAs, Fernando de (2018), La Celestina de Fernando de Rojas sale de nuevo a la luz en facsímil (Zaragoza, 1554), edición a expensas y al cuidado de Alfonso Fernández González, Zaragoza - Sabadell, Establecimiento Tipográfico Francesc Fusté Serena.

RoJAS, Fernando de, y antiguo autor (2000), La Celestina: tragicomedia de Calisto y Melibea, ed. Francisco Lobera et al., Barcelona, Crítica.

Soтневу's (1978), Spanish Books Printed in the Spanish Netherlands Formed by the Late J. Peeters-Fontainas, Sold by Order of the Family, London, Sotheby's.

SWB, «Südwestdeutscher Bibliotheksverbund», catálogo colectivo. Accedido el 01 de noviembre de 2020, disponible on-line <https://www. bsz-bw.de>.

UNICAT, "Union Catalogue of Belgian Libraries», catálogo colectivo. Accedido el 01 de noviembre de 2020, disponible on-line <https://www. unicat.be/>.

USTC, «Universal Short Title Catalogue», base de datos bibliográfica. Accedido el 01 de noviembre de 2020, disponible on-line <https://www. ustc.ac.uk>.

Vega García-Luengos, Germán et al. (2012), Ediciones de teatro español en la Biblioteca de Menéndez Pelayo: (hasta 1833). Vol. 1. A-D, 1-1401, Alicante, Biblioteca Virtual Miguel de Cervantes, 2012, edición digital a partir del original de Kassel, Reichenberger, 2001. Accedido el 01 de noviembre de 2020, disponible on-line <http://www.cervantesvirtual. com/nd/ark:/59851/bmc805q1>.

Whitney, James Lyman (1879), Catalogue of the Spanish Library and of the Portuguese Books Bequeathed by George Ticknor to the Boston Public Library: Together with the Collection of Spanish and Portuguese Literature in the General Library, Boston, Printed by order of the Board of Trustees. 
